\title{
Serum IgG Profiling of Toddlers Reveals a Subgroup with Elevated Seropositive Antibodies to Viruses Correlating with Increased Vaccine and Autoantigen Responses
}

\author{
Patricia Pichilingue-Reto ${ }^{1,2,3}$ • Prithvi Raj ${ }^{1}$. Quan-Zhen $\mathrm{Li}^{1}$ • Igor Dozmorov ${ }^{1}$ - David R. Karp ${ }^{4}$ • Edward K. Wakeland ${ }^{1}$. \\ Morgan Nelson $^{5} \cdot$ Rebecca S. Gruchalla $^{2,5} \cdot$ M. Teresa de la Morena ${ }^{6} \cdot$ Nicolai S. C. van Oers $^{1,2,7}$ (D)
}

Received: 12 September 2020 / Accepted: 10 February 2021 / Published online: 3 March 2021

(C) The Author(s), under exclusive licence to Springer Science+Business Media, LLC part of Springer Nature 2021

\begin{abstract}
Purpose The human antibody repertoire forms in response to infections, the microbiome, vaccinations, and environmental exposures. The specificity of such antibody responses was compared among a cohort of toddlers to identify differences between seropositive versus seronegative responses.

Methods An assessment of the serum IgM and IgG antibody reactivities in 197 toddlers of 1- and 2-years of age was performed with a microfluidic array containing 110 distinct antigens. Longitudinal profiling was done from years 1 to 2 . Seropositivity to RNA and DNA viruses; bacteria; live attenuated, inactive, and subunit vaccines; and autoantigens was compared. A stratification was developed based on quantitative variations in the $\operatorname{IgG}$ responses. Clinical presentations and previously known genetic risk alleles for various immune system conditions were investigated in relation to $\operatorname{IgG}$ responses.

Results IgG reactivities stratified toddlers into low, moderate, and high responder groups. The high group (17\%) had elevated IgG responses to multiple RNA and DNA viruses (e.g., respiratory syncytial virus, Epstein-Barr virus, adenovirus, Coxsackievirus) and this correlated with increased responses to live attenuated viral vaccines and certain autoantigens. This high group was more likely to be associated with gestational diabetes and an older age. Genetic analyses identified polymorphisms in the IL2RB, TNFSF4, and INS genes in two high responder individuals that were associated with their elevated cytokine levels and clinical history of eczema and asthma.

Conclusion Serum IgG profiling of toddlers reveals correlations between the magnitude of the antibody responses towards viruses, live attenuated vaccines, and certain autoantigens. A low responder group had much weaker responses overall, including against vaccines. The serum antibody screen also identifies individuals with IgG responses to less common infections (West Nile virus, parvovirus, tuberculosis). The characterization of the antibody responses in combination with the identification of genetic risk alleles provides an opportunity to identify children with increased risk of clinical disease.
\end{abstract}

Keywords IgGs $\cdot$ serum antibody responses $\cdot$ antigen arrays $\cdot$ toddler immune responses $\cdot$ vaccines

Patricia Pichilingue-Reto and Prithvi Raj contributed equally to this work.

Nicolai S. C. van Oers

Nicolai.vanoers@utsouthwestern.edu

1 Department of Immunology, The University of Texas Southwestern Medical Center, NA2.200, 6000 Harry Hines Blvd., Dallas, TX 75390-9093, USA

2 Department of Pediatrics, The University of Texas Southwestern Medical Center, NA2.200, 6000 Harry Hines Blvd., Dallas, TX 75390-9093, USA

3 Department of Infectious Diseases, St. Jude Children's Research Hospital, Memphis, TN 38105, USA
4 Department of Internal Medicine, The University of Texas Southwestern Medical Center, NA2.200, 6000 Harry Hines Blvd., Dallas, TX 75390-9093, USA

5 Children's Health, Dallas, TX 75235, USA

6 Department of Pediatrics, Division of Immunology, University of Washington and Seattle Children's Hospital, Seattle, WA 98105, USA

7 Department of Microbiology, The University of Texas Southwestern Medical Center, NA2.200, 6000 Harry Hines Blvd., Dallas, TX 75390-9093, USA 


\section{Introduction}

Infants have limited amounts of their own serum immunoglobulins (Igs, antibodies) at birth, relying on the transplacental transfer of maternal IgG and oral intake of IgA from breast milk for protection against infections. Such maternal antibodies provide a temporary defense against infections. During their first years of life, toddlers/children begin producing a circulating pool of immunoglobulins in response to infections, vaccinations, the developing microbiome, and diverse environmental exposures [1-4]. Best defined in mice, there are two major types of B cells, conventional B-2 cells and B-1 cells, that secrete the immunoglobulins. In humans, the B-1 equivalent populations are defined with the markers $\mathrm{CD} 19^{+} \mathrm{CD} 20^{+} \mathrm{CD} 27^{+} \mathrm{CD} 38^{\text {low } / \text { int }} \mathrm{CD} 43^{+}$. Such B-1 cells are more predominant at birth, primarily secreting low-affinity IgM isotype antibodies [5, 6]. As an infant grows, the conventional B-2 cells form the major antibody-secreting population [6-8]. Expressing terminal deoxynucleotide transferase (TdT), these B-2 cells generate the various antibody isotypes (IgM, IgG, IgA, IgE, IgD). These antibodies have higher affinities for antigens, which occurs via somatic hypermutation of the antigen-binding complementarity determining regions [9]. Importantly, even young babies can generate high-affinity neutralizing antibodies towards various pathogens. For both human immunodeficiency virus (HIV) type 1 and malarial infections, somatically hyper-mutated high-affinity $\operatorname{IgG}$ is already evident in infants by 3 months of age $[10,11]$. The $B$ cell populations secreting these antibodies will eventually form a pool of memory B cells. This is consistent with the observation that the overall memory B cell numbers are very low at birth, with about $50 \%$ of all circulating B cells considered immature $[7,8]$.

By 1 year of age, a toddler produces most of their own circulating antibodies, with maternally derived IgG catabolized due to the 5-6 week half-live of the protein [12-14]. Maternal antibodies can have longer half-lives (> 80 days), revealed through the presence of vaccine-specific antibodies present in unvaccinated infants [15-17]. In a 1-year-old, the IgG levels are approximately $70 \%$ of adult levels, while IgA is only at $30 \%$ values. While most infants produce sufficient levels of Igs for protection against infections, several clinical conditions exist wherein these are inadequate. One more common clinical condition is transient hypogammaglobulinemia of infancy, wherein an infant produces insufficient quantities of protective antibodies against pathogens. This condition can extend into the 3rd year of life, leading to more infections [18]. In addition, there are infants who have inborn errors of immunity that impede antibody production [19].

Given the importance of antibodies in protecting individuals against infections and the critical role of vaccines in directing this antibody response, little has been reported about the variations that may exist in such responses among a cohort of toddlers/children. To address this, we performed a comparative analysis of the serum IgM and IgG antibody specificities among normal 1- and 2-year-old toddlers towards a panel of 110 distinct antigens. These included antigens used to monitor immunoglobulin responses to common viral and bacterial infections along with vaccine antigens, autoantigens, and allergens. The screen was performed with microfluidic antigen arrays, which permits a screening of the antibody reactivities to 110 distinct antigens simultaneously. Such arrays have a greater sensitivity than conventional ELISA and are excellent for comparative analyses with a broad range of antigens [20]. Our findings indicate that the majority of toddlers/children have overall low $\operatorname{IgG}$ reactivities to the various antigens. However, $17 \%$ of the cohort exhibited elevated IgG responses to specific RNA and DNA viruses, several live attenuated viruses, and a few autoantigens. A DNA sequence analysis with several of the individuals in the cohort revealed particular genetic polymorphisms (IL2RB, TNFSF4, and INS) that correlated with higher IgG responses and was associated with two individuals who had elevated circulating cytokine levels. Taken together, these findings suggest normal toddlers/ children have very divergent antibody responses in their first 2 years of life, with a subset exhibiting strong seropositive responses correlating with more effective vaccine responses as well as increased autoantibody levels.

\section{Methods}

\section{Study Approval}

The Institutional Review Board at UT Southwestern Medical Center approved this study (IRB\# 072010-009 and IRB\# 112010-013). Parental consent was obtained for 200 individuals, and 3 withdrew over the course of the sampling. The remaining 197 were used for the analyses described in the current manuscript. All toddlers who participated in this study were seen by pediatricians for their standard of care wellness visits. The pediatricians were affiliated with Children's Health Pediatric Group at Children's Health, Dallas, Texas (Medical District Site). Blood samples were obtained following standard of care draws for hemoglobin and lead levels, which is mandated by the State of Texas for toddlers and children at the ages of 1 and 2. A total of 122 blood draws were from 1-yearold toddlers and 116 from 2-year-old children. Forty-one of these Yr.2 samples were biological replicates from those individuals who had returned after their 1st-year wellness visits clinical information including birth status, age, sex, ethnicity, growth, vaccination dates, antibiotic and/or anti-viral use, drug treatments, and other healthrelated information was compiled (Table 1). 
Table 1 Clinical information from 1971 and 2-year-old toddlers. Most subjects showed no pertinent antecedents. Relevant past medical history compiled included asthma and allergic conditions

\begin{tabular}{|c|c|c|}
\hline & & $n(\%)$ \\
\hline \multirow[t]{2}{*}{ Gender } & Male & $92(47)$ \\
\hline & Female & $105(53)$ \\
\hline \multirow[t]{2}{*}{ Age } & 12 months & $122(62)$ \\
\hline & 24 months & $75(38)$ \\
\hline \multirow[t]{3}{*}{ Race/ethnicity } & Hispanic & $117(59)$ \\
\hline & African American & $63(32)$ \\
\hline & White/other & $17(9)$ \\
\hline \multirow[t]{2}{*}{ Term birth } & Full term & $174(88)$ \\
\hline & Premature & $23(12)$ \\
\hline \multirow[t]{2}{*}{ Delivery } & Vaginal & $119(60)$ \\
\hline & C-section & $78(40)$ \\
\hline \multirow[t]{2}{*}{ Feeding History } & Breast milk & $139(71)$ \\
\hline & Formula & $58(29)$ \\
\hline \multirow[t]{2}{*}{ Gestational diabetes } & Yes & $14(7)$ \\
\hline & No & $183(93)$ \\
\hline \multicolumn{3}{|c|}{ Pertinent medical history } \\
\hline \multirow[t]{4}{*}{ Allergic } & Eczema & $23(12)$ \\
\hline & Allergic rhinitis & $9(5)$ \\
\hline & Asthma/RAD ${ }^{\mathrm{a}}$ & $11(6)$ \\
\hline & Food allergy & $2(1)$ \\
\hline \multirow[t]{2}{*}{ Gastrointestinal } & GERD $^{b}$ & $10(5)$ \\
\hline & Constipation & $4(2)$ \\
\hline Cardiovascular & Heart disease & $9(5)$ \\
\hline \multirow[t]{2}{*}{ Neurological } & Seizure disorder & $2(1)$ \\
\hline & Developmental delay & $18(9)$ \\
\hline \multirow[t]{3}{*}{ Nutritional } & Obesity & $4(2)$ \\
\hline & Underweight & $3(2)$ \\
\hline & Anemia & $26(13)$ \\
\hline \multirow[t]{2}{*}{ Vaccination status } & Up-to-date & $133(68)$ \\
\hline & Not up-to-date & $64(32)$ \\
\hline \multirow[t]{2}{*}{ Use of antimicrobials } & Yes & $107(54)$ \\
\hline & No & $90(46)$ \\
\hline \multirow[t]{3}{*}{ Family history } & Diabetes & $59(30)$ \\
\hline & Asthma & $36(18)$ \\
\hline & Autoimmune diseases ${ }^{c}$ & $7(4)$ \\
\hline
\end{tabular}

${ }^{\mathrm{a}} R A D$, reactive airway disease

${ }^{\mathrm{b}} G E R D$, gastroesophageal reflux

${ }^{c}$ Autoimmune diseases reported in the family include rheumatoid arthritis, Hashimoto disease, or autoimmune thyroiditis and SLE

\section{Blood Sampling and Processing}

Between 30 and $300 \mu \mathrm{l}$ of blood was collected from each individual using serum blood collection tubes (Item \#077151, ThermoFisher Scientific). This meant that for most individuals, only a finger stick was required for blood sampling.
After coagulation, serum was separated from blood cells following 10-min centrifugation at 10,000 rpm.

\section{Antigen Array Analysis}

Serum IgG and IgM reactivity were tested with a microfluidic antigen array comprising 110 antigens, including 53 autoantigens, 26 pathogen agents, 17 vaccine antigens, and 14 allergens along with two controls (HEL, BSA) (Supplemental Fig. 1a). Serially diluted human IgG and IgM were added as internal controls. In addition, serially diluted anti-human $\operatorname{IgG}$ was added in 4 additional wells to calculate the relative serum IgG levels. This customized antigen panel was named Kids Panel I and is available from the Microarray and Immunophenotyping Core Facility at the University of Texas Southwestern Medical Center. The 110 antigens were printed in duplicate on nitrocellulose membrane-coated slides. Each slide can be used with 16 samples, 15 different serum samples were compared in one slide along with a PBS control $[21,22]$. Such microfluidic antigen arrays were developed as previously described $[22,23]$. In brief, $1 \mu$ of serum was diluted 1:100 in PBS with 0.01\% Tween 20 and applied to the slide array using microfluidic applications. After washing, IgG and IgM isotype antibodies binding to the diverse antigens were detected with Cy-3-conjugated anti-human IgG (1:1000) and Cy-5-conjugated anti-human IgM (1:1000) secondary antibodies (Jackson ImmunoResearch Laboratory). Array slides were scanned with GenePix 4400A scanner (Molecular Devices) to generate images for each array. This was converted to Genepix Report file (GPR) using Genepix Pro7.0 software (Molecular Devices). The averaged fluorescent signal intensity of each antigen was subtracted by the local background and the PBS control signal. The net fluorescent intensity of each antigen was further normalized with internal controls to generate the normalized fluorescent intensity (nfi) values. The normalization procedure was used to exclude variations due to differences in the processing, labeling, and fluorescence levels between differing runs. To control for variations with different slides prepared over 3 years (samples processed at different times), the 4 dilutions of the IgG control were analyzed at $1(1 \mathrm{mg} / \mathrm{ml}), 1 / 2(0.5 \mathrm{mg} / \mathrm{ml}), 1 / 4$ $(0.25 \mathrm{mg} / \mathrm{ml})$, and $1 / 8(0.125 \mathrm{mg} / \mathrm{ml})$ dilutions. This was done to identify the linear range of the values. The averaged $\operatorname{IgG}$ control was then calculated using the formula $A i j$, where $j$ is the run number and $i$ is the sample number within the order. This parameter was calculated for each sample as $\left(\operatorname{IgG}_{1}+2 \times\right.$ $\left.\operatorname{IgG}_{1 / 2}+4 \times \operatorname{IgG}_{1 / 4}\right) / 3$, where $\operatorname{IgG}_{\mathrm{t}}$ was the dilution of control $\mathrm{IgG}$ for a given sample. All other nfi values were adjusted based on these normalized IgG controls. After this adjustment, the corrected $\mathrm{IgG}$ controls for each order were equalized. Individual sample adjustments were achieved by the use of individual corrected controls. A non-supervised clustering was created for the initial analysis of the nfi levels. This was 
based on the Pearson correlation as described in detail elsewhere [24]. The appropriate threshold for the correlation was calculated using a simulation study. Responses to specific antigens were sorted by their relationships and the clustering process was started with the higher nfis, separated into different categories (pathogens, vaccines, autoantigens, allergens). This is an iterative process as described previously [25-28]. For the heat map presentations, a distinct standardization procedure was used. For each nfi for a particular antigen, the maximal level was selected and divided by all the other nfis. This allowed all samples with the same antigen target to appear as a range from 0 to 1 . To discriminate low and high expressions in a visual presentation by color (green-low; red-high), a border (0.2) was defined. After that, the nfis after the first step of standardization above 0.2 were presented in red colors, whereas low expression below 0.2 transformed to a negative value and presented in green colors.

\section{ELISA Assays and Serum Cytokine Measurements}

ELISA assays were used to qualitatively identify human serum IgG antibody reactions to Respiratory Syncytial Virus (Abcam Inc. Cambridge, MA) along with cytomegalovirus $(\mathrm{CMV})$ and Epstein-Barr virus (EBV) (Zeus Scientific, Inc. Branchburg, NJ). These assays are used to identify patients with serologic evidence of previous or primary infections. Anti-nuclear antibody responses (ANA) were assayed using a commercially available kit (QUANTA Lite ANA ELISA, Inova Diagnostics, San Diego, CA). For serum cytokine measurements, a Bio-Plex Pro Human Cytokine 27-plex Assay was performed in the Genomics and Immunophenotyping Core facility at UT Southwestern Medical Center according to the manufacturers' instructions.

\section{Genotyping}

Blood cells were used for DNA isolation. The DNA extraction was performed with QIAmp DNA Mini Kit from QIAGEN. About $250 \mathrm{ng}$ of genomic DNA was used to genotype markers with Infinium ImmunoArray-24 v2 BeadChip from Illumina. The genome-wide content on this array is based on information on the populations from the 1000 Genomes Project. The markers tiled on array include variants with established disease associations, relevant pharmacogenomics markers, and curated exonic content based on ClinVar, NHGRI, PharmGKB, and ExAC databases. QC content enables sample identification and tracking for large-scale genomics and screening applications. The image files from the scanner were imported into Genome Studio, Illumina. Marker genotypes were called using the genotyping module in GenomeStudio.

\section{Statistics}

Statistical analyses were performed using GraphPad Prism software version 9.2 (GraphPad). A $p$ value of less than 0.05 was considered statistically significant. ns denotes not significant. For figures, standard one-way ANOVA was used in most cases (assumes equal variances), unless distinct tests were used as indicated in the figure legends. For Fig. $6 \mathrm{c}$, d, a normal oneway ANOVA analysis was applied. Genetic association analysis was performed using the SVS Golden Helix program.

\section{Results}

\section{The Magnitude of the Serum IgG Antibody Reactivities in Toddlers/Children Reveals Subgroups with Low, Moderate, and High Responses to Pathogen, Vaccine, and Autoantigens}

The range of binding specificities of the circulating serum antibodies among toddlers/children towards diverse infectious agents, vaccines, and autoantigens has not been well defined. To address this, we tested serum from 197 toddlers/ children with an antibody profiling screen (Fig. 1a). The demographics of 197 individuals are provided in Table 1. Most of the subjects were recruited at 1 year of age (62\%). Fifty-three percent of the cohorts were female. The ethnic distribution of the cohort was 59\% Hispanic, 32\% African American, and 9\% White or Asian. The majority of toddlers (91\%) were born full term, with $60 \%$ born via spontaneous vaginal delivery. Seventy-one percent were breastfed, either exclusively or mixed with formula. Maternal gestational diabetes was reported for $7 \%$ of the subjects. One hundred thirty-three $(68 \%)$ were upto-date with vaccinations. One hundred eight subjects (55\%) did not have any pertinent past medical history, while $13 \%$ had anemia, 12\% had eczema, 9\% had developmental delay, and $6 \%$ had asthma, among other clinical conditions. Up to $54 \%$ received antibiotics at least once (electronic medical records, EMR). In regard to family history, $30 \%$ had a family history of diabetes, $18 \%$ reported a family history of asthma while $4 \%$ family history of diagnosis of autoimmune diseases including rheumatoid arthritis, Hashimoto disease, or autoimmune thyroiditis and systemic lupus erythematosus (SLE). Blood samples were obtained from the 1- and 2-year-old toddlers/children who were being seen for standard of care health visits including lead and hemoglobin screens. Serum was separated from the cellular constituents and used for the antibody profiling, with the pelleted cellular material used for DNA isolation and subsequent genomic studies (Fig. 1a).

The $\operatorname{IgM}$ and $\operatorname{IgG}$ reactivities were tested with 238 serum samples, 41 of which corresponded to toddlers who had second blood draws a year (Yr.2) after their initial Yr.1 sampling along with multiple technical replicates (Fig. 1b, Supplemental Fig. 
a

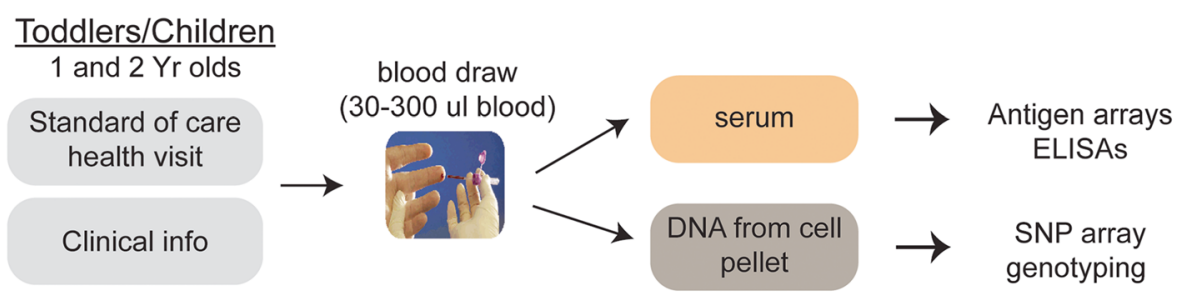

b

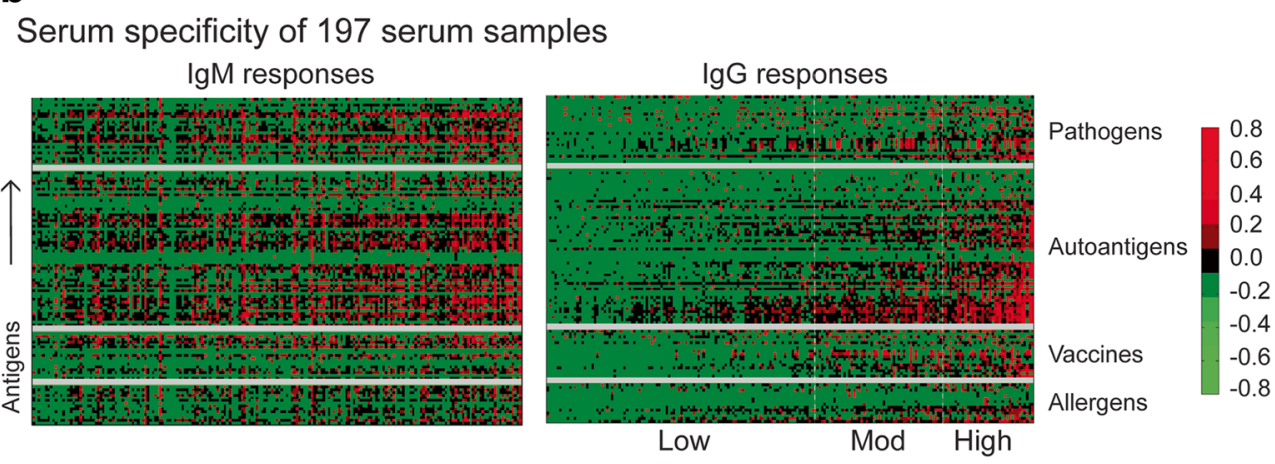

C

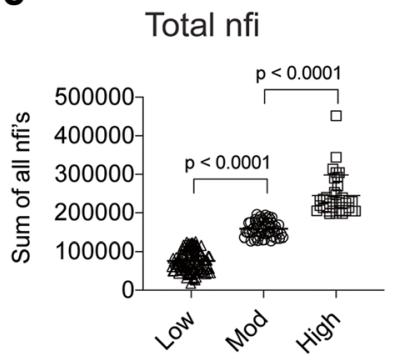

d
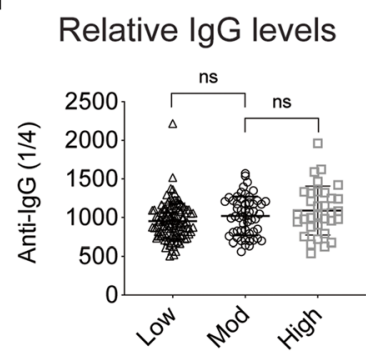

e

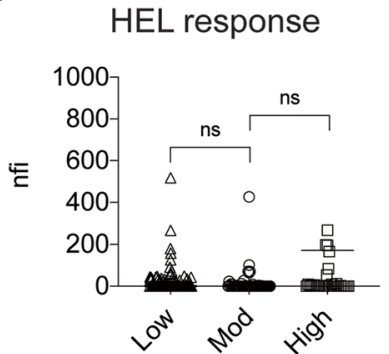

Fig. 1 The serum IgG specificities of toddlers/children reveal distinct subgroup responses to pathogens, autoantigens, vaccines, and allergens. a Schematic of the immune response profiling of 1- and 2-year-old toddlers. Blood samples are processed for serum and cellular DNA, used for antigen arrays, ELISA assays, and DNA sequencing. Medical records are reviewed along with family history. b The antigen array was used to assess the antibody responses in a cohort of 1- and 2-year toddlers/children. One hundred ninety-seven individual serum samples were tested against an antigen array comprising 110 antigens (detailed in the Methods section). The heat maps, with IgM on the left and IgG on the right, correspond to 197 samples; each column is 1 sample/toddler. The various categories of antigens are indicated. The heat map indicates positive antibody reactivity (red color) or minimal to no reactivity (green color). $\mathbf{c}$ The sum of the IgG nfi responses for every antigen was calculated, revealing a segregation of the cohort into low, moderate (Mod), and high reactivity groups. d The relative serum IgG level for each individual, stratified into the low, Mod, and high response group, was determined. e The nfi levels for hen egg lysozyme (HEL), used as a control antigen, were determined for each individual in the low, Mod, and high response groups. Statistical analyses were done with one-way ANOVA using Tukey's correlation, and $p$ values shown. ns, not significant

antigens (Fig. 1b, green). To compare the responses, a stratification was developed based on the overall levels of the normalized fluorescence intensities (nfi). As the nfis ranged from values as low as 0 to as high as 65,000 for different samples, the average sum and standard deviation of the nfis towards all 110 antigens was calculated $(126,051 \pm 70,839)$. Toddlers with a sum of nfi value $1 \mathrm{SD}$ above the mean were classified as high responders $(n=33)$ while those $1 \mathrm{SD}$ below were designated as low responders $(n=110)$. The group with nfi values in between these ranges were designated as moderate (Mod) responders $(n=54)$ (Fig. 1c). The distribution revealed that 56\%, 27\%, and $17 \%$ of the cohort were low, Mod, and high responders, respectively (Fig. 1b, c). This low, Mod, and high classification 
was not due to differences in the overall IgG levels as no statistically significant differences were noted when comparing the 3 subgroups (Fig. 1d). The serum $\mathrm{IgG}$ reactivities among the high response group were also not due to high basal increases in IgG reactivities as 196/197 individuals had similar background level responses to HEL and other antigens (Fig. 1e, Supplemental Excel File 1). Concerning clinical conditions, pertinent medical history was compared in the low, Mod, and high groups (Supplemental Table S2). There were no specific conditions particular to the low, Mod, or high groups. Taken together, these results reveal a marked distinction in the serum IgG responses among 1- and 2-year-old toddlers and identify a subset with elevated $\operatorname{IgG}$ reactivities to many antigens.

\section{Identification of Particular Viral Infectious Exposures in Toddlers/Children with Microfluidic Antigen Arrays}

The microfluidic antigen array enables one to identify toddlers/ children with seropositive IgG responses to many distinct infectious agents. We assessed the responses to respiratory syncytial virus (RSV) and cytomegalovirus (CMV). Serum IgG nfi levels were compared with two RSV antigens (RSV and an RSV glycoprotein) and two cytomegalovirus antigens (CMV and CMV-M). Two distinct antigens were used to cover more epitopes for each virus. The responses were plotted against the low, Mod, and high nfi stratified groups (Fig. 2a, b). RSVspecific IgG responses clustered among the high response group (Fig. 2a). CMV seropositive individuals were distributed throughout the low, Mod, and high response groups (Fig. 2b). Some individuals had high IgG nfis to both antigens, as revealed with overlapping red/black peaks in the graph (Fig. 2a, b). To establish whether these seropositive responses were clinically informative, an independent serologic ELISA assay was used to confirm previous or primary respiratory syncytial virus (RSV) or cytomegalovirus (CMV) infections for some of the toddlers (Fig. 2a, b, open circles). Positivity defined by an ELISA for the RSV glycoprotein revealed that a minimum of 15,000 nfi units would identify individuals with RSV seropositivity (Fig. 2a, y-axis on right). Pearson's correlation coefficients revealed a high correlation $\left(r=0.903, R^{2}=0.816\right)$ of statistical significance between the microfluidic-based nfis and the ELISA (Supplemental Fig. 2a, $p<0.0001$ ). Based on this defined nfi cutoff, 31 out of 197 toddlers likely had previous or primary RSV infections targeting the glycoprotein. Of note, the second RSV antigen used for the microfluidic array suggested an even higher prevalence as not all individuals targeted both antigens. For CMV seropositivity, an ELISA assay was performed for $>20$ individuals who had either low or high nfis towards a CMV antigen. Pearson's correlation coefficients revealed a high correlation $\left(r=0.876, R^{2}=0.768\right)$ with statistical significance $(p<0.0001)$ (Supplemental Fig. $2 b)$. The ELISA positivity value suggested that a nfi threshold of 600 is indicative of previous or primary CMV infections (Fig. 2b).
Twenty out of 197 individuals were CMV seropositive. We subsequently compared the patterns of seropositivity for 6 additional viral infections: adenovirus, coronavirus, Coxsackievirus, Epstein-Barr virus (EBV), echovirus type 9, and enterovirus 71 (Fig. 2c-h). While ELISAs were not performed against all the viruses due to limited sample volumes for some of the toddlers, there were clearly demarcated IgG nfi values above the normal. Using this analysis in combination with known frequencies of particular viral infections in the 12-year-old age group, a nfi cutoff of positivity was established for each virus. The overall comparative results revealed a clustering of seropositivity among the high responder group for 6 of 8 viruses, the exceptions being CMV and coronavirus (Fig. 2ah).

\section{A Subset of Toddlers Has Strong IgG Antibody Reactivities to Selected Pathogens and Live Attenuated Viruses}

The high response cohort of toddlers had elevated IgG nfi responses to 6 different viruses relative to the low and Mod responders (Fig. 2a-h). To examine whether this pattern was statistically significant, the nfi values detected for the various antigens were plotted against the low, Mod, and high response groups. The high responder group had more pronounced IgG nfi values against 7 RNA and DNA viruses of statistical significance (Fig. 3a). For CMV, we used the CMV type III antigen, with seropositivity better reflecting a recent or active viral infection [30]. The elevated nfi response among the high responder group was not against every virus, as noted for a coronavirus (Fig. 2a, c, 3a, Supplemental Fig. 3a). Another example of a more evenly distributed response among the groups was that against molluscum contagiosum virus. About $41 \%$ of all the toddlers/children screened had IgG responses towards this virus (> $5000 \mathrm{nfi}$ values) that was spread across the 3 groups (Fig. $3 a)$. In addition to viral pathogens, the high responder group had elevated nfis towards selected bacterial antigens and parasites (Pseudomonas fluorescens lipase and toxoplasma) (Fig. 3a). Individuals with seropositive responses to less common infections were identified, including some with elevated IgG nfis to West Nile virus and parvovirus (Supplemental Fig. 2). Three toddlers had reactivity to the Mycobacterium tuberculosis antigen early secretory antigenic target (ESAT6), suggesting possible exposures to mycobacteria (Fig. 3a).

We next compared the IgG responses among the low, Mod, and high groups to routine childhood vaccines. The high responder group had statistically significant elevated nfis against the live attenuated viruses targeting mumps, rotavirus, and varicella-zoster (Fig. 3b, Supplemental Fig. 3b). For most inactive or subunit vaccines, there were no statistically significant differences in the 3 groups. This can be seen with the similar serum IgG responses to influenza, hepatitis $\mathrm{B}$, and Streptococcus pneumoniae vaccines among the low, Mod, 
Fig. 2 Serum IgG nfi seropositive responses to particular RNA and DNA viruses cluster among the high responder group. a The relative $\mathrm{IgG}$ responses, measured using the nfi values from the microarray analysis, are shown for two distinct RSV antigens, shown as black (RSV) and red lines (RSV glycoprotein, RSV$\mathrm{Gp).} \mathrm{Each} \mathrm{line} \mathrm{corresponds} \mathrm{to} \mathrm{an}$ individual sample, ranged from the lowest to highest overall nfi value. The separation among the low, Mod, and high groups is shown below the y-axis. Serum IgG responses, determined using an independent ELISA assay for an RSV-Gp, are shown for a subset of toddlers. The adjusted $\mathrm{OD}_{450}$ values are shown as open circles. b The relative IgG nfi responses are shown for two distinct CMV antigens, shown with black (CMV) and red lines (CMV-M). An independent ELISA assay for the CMV antigen was performed using samples from a subset of toddlers. The open circles reveal the adjusted $\mathrm{OD}_{450}$. $\mathbf{c}-\mathbf{h}$ Serum IgG nfi values specific for $\mathbf{c}$ adenovirus, $\mathbf{d}$ coronavirus, e Coxsackievirus, $\mathbf{f}$ echovirus type 9, and $\mathbf{f}$ enterovirus 71 are shown, plotted against the increasing overall nfis calculated for each individual. Values above the dotted lines in the graphs suggest infection-based seropositivity a

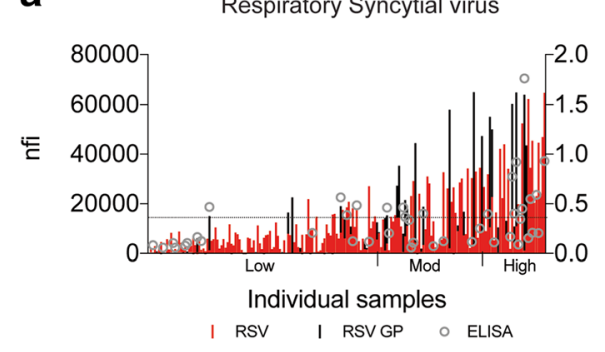

C

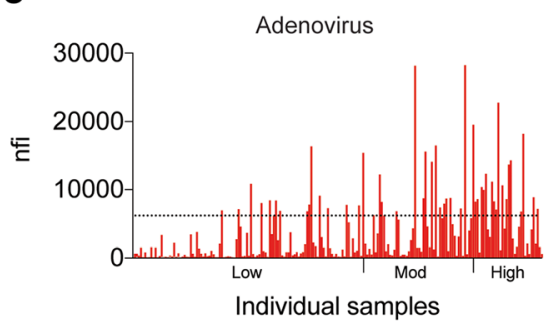

e

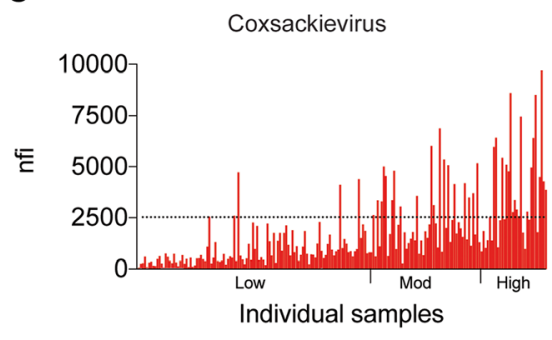

g

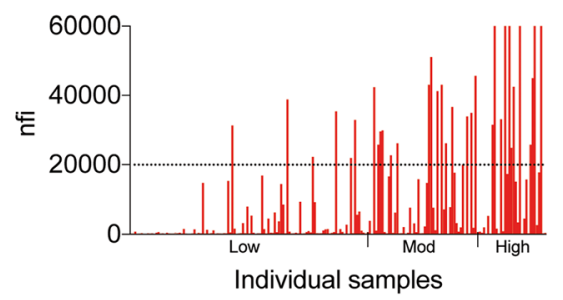

b

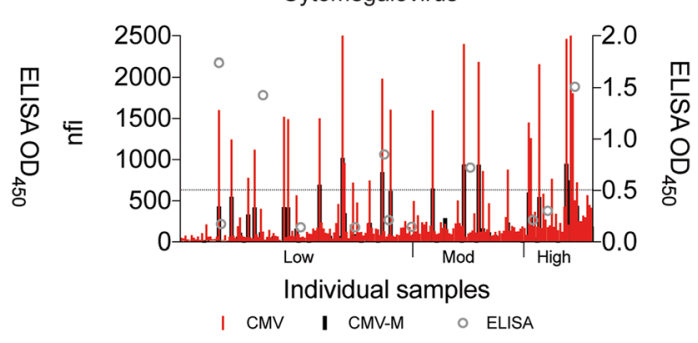

d

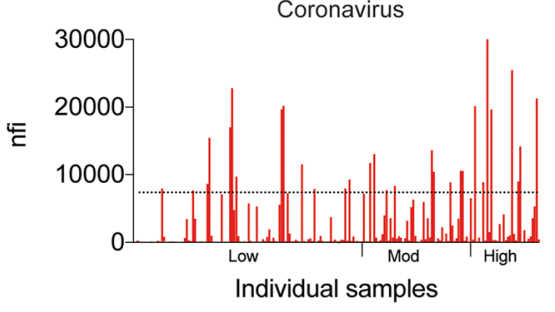

f

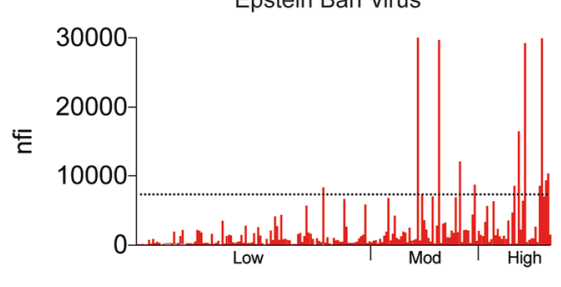

Individual samples

h

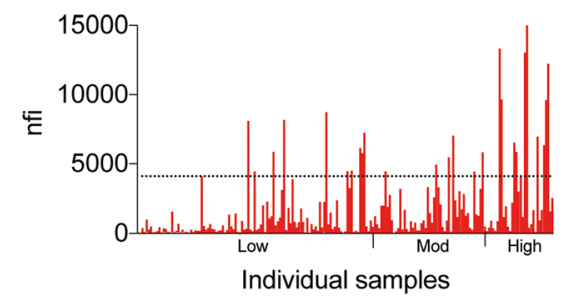

and high groups (Fig. 3b). Only with the low responder group were slightly diminished nfis of statistical significance noted towards a few inactivated or subunit vaccines, such as hepatitis A and tetanus toxoid (Fig. 3b). Taken together, our results suggest that individuals with enhanced IgG nfi values towards numerous DNA and RNA viruses tend to have elevated seropositive IgG responses to live attenuated vaccines, which were primarily against viruses such as measles, mumps, and rubella.

Published reports with adult cohorts indicate a positive correlation between $\mathrm{IgG}$ responses to viruses and to self-antigens [31]. We addressed whether this was also apparent in toddlers/ children by first comparing the low, Mod, and high response groups against 53 different autoantigens. The high responder group had statistically significantly higher IgG nfi values to a variety of autoantigens, including nucleosome components, arrestin beta 1, a histone mix, endothelial lysate, Smith antigens, and others (Fig. 4a). There were additional increased nfi values against defined autoantigens in the high response group of statistical significance (Supplemental Fig. 4). Elevated nfi values towards the autoantigens were still much lower than that noted for a cohort of adult SLE patients, and none of the toddlers/ children screened had signs of autoimmunity (Supplemental Fig. 5). Also, the high response cohort had limited IgG reactivities to the bulk of autoantigens screened. A longitudinal analysis will be necessary to determine if the high response group has an increased susceptibility to autoimmune diseases that may present later in life. Another measure of potential autoimmunity is the formation of antibodies against nuclear antigens (ANA) [32]. ANA values were determined using an ELISA- 

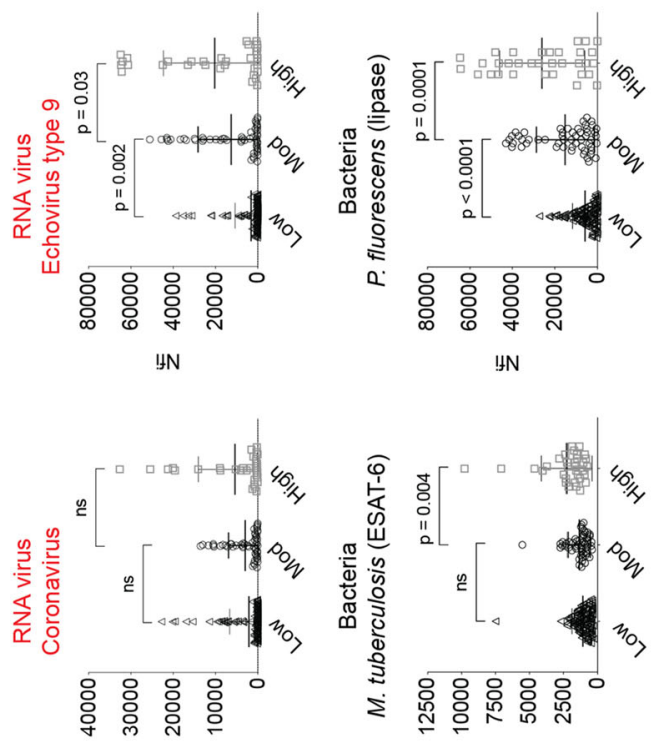

! $N$

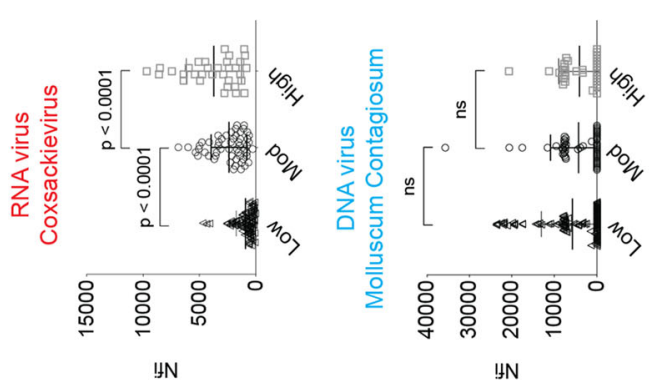

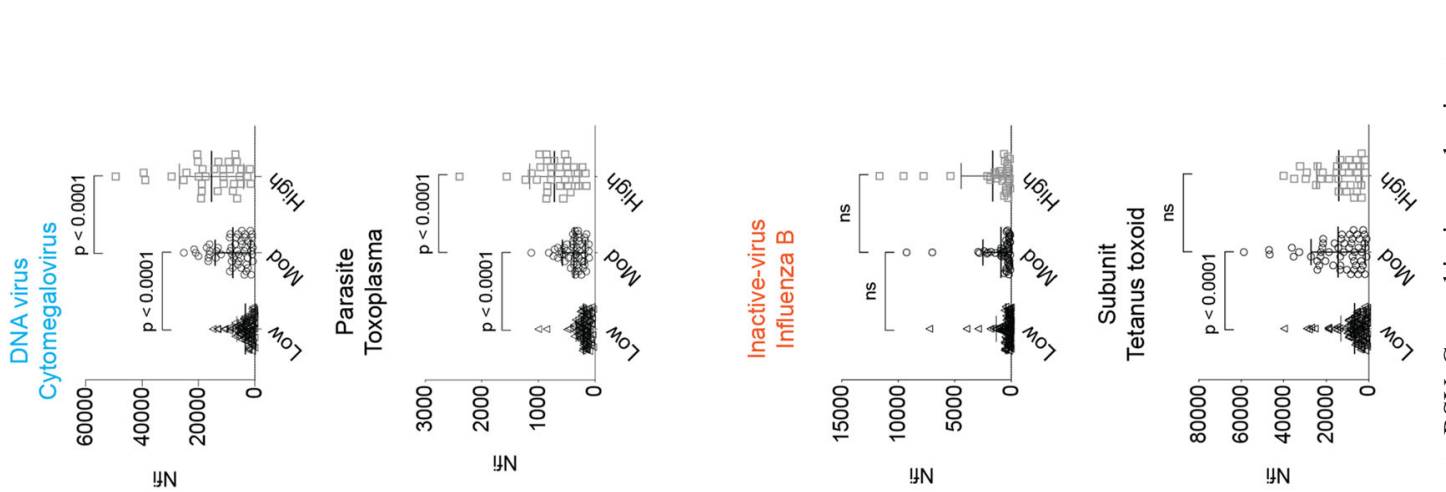

总总蒙表若

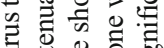
击 워용 过

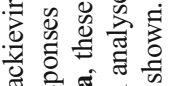
论 80 会造语

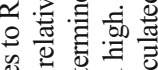
递察

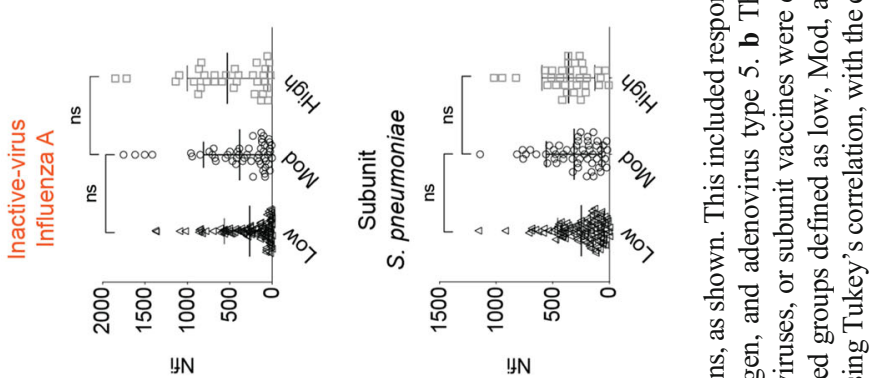
공.

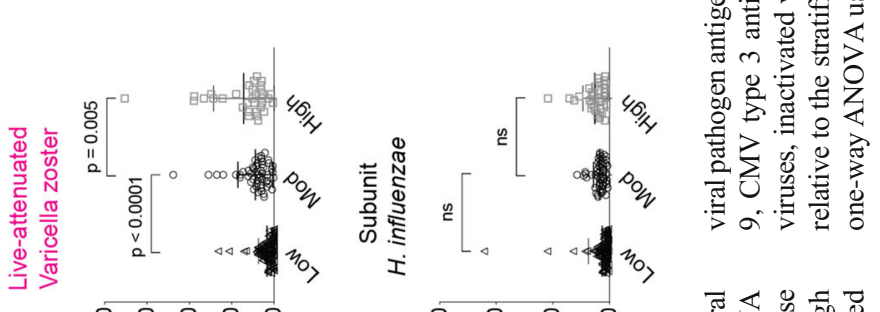

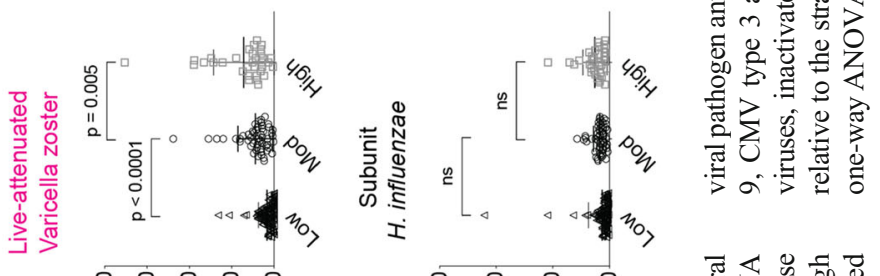
영 웅 웡 웡 IN ㅇํㅇ 응

픈

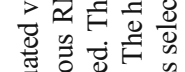

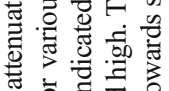

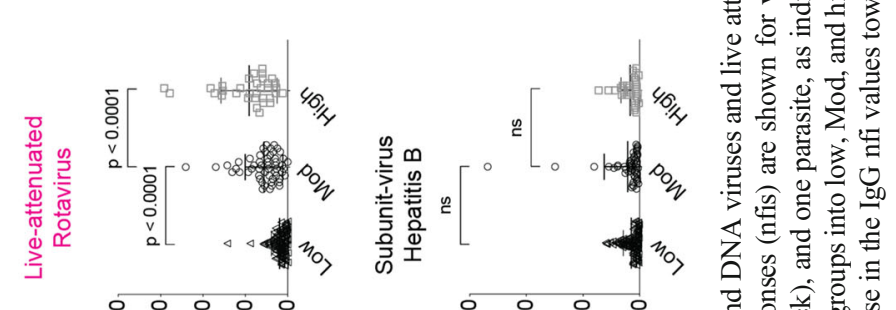

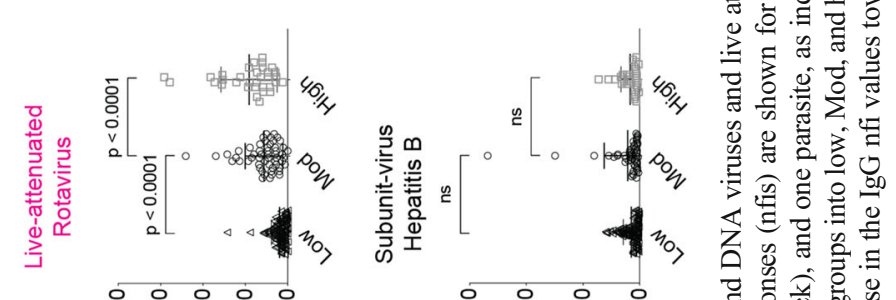

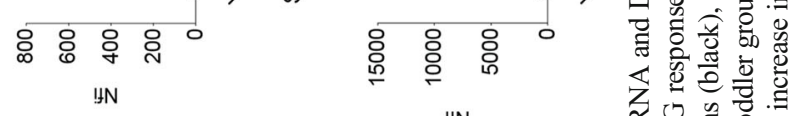
IN $\quad$ 至

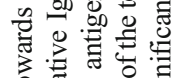

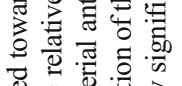

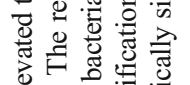

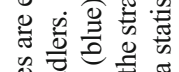

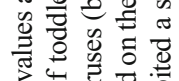

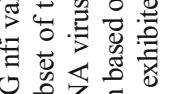

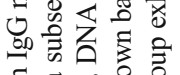

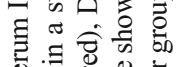

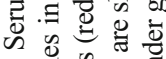

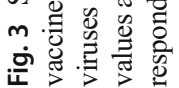




\section{a}

\section{Autoantigens}

Nucleosome

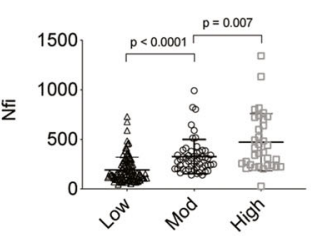

b

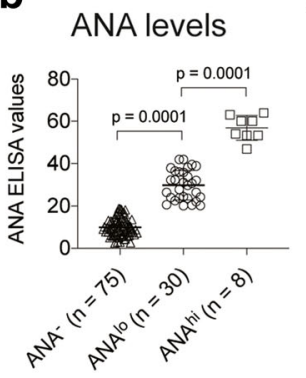

Arrestin beta 1

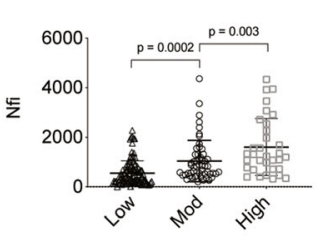

Histone mix

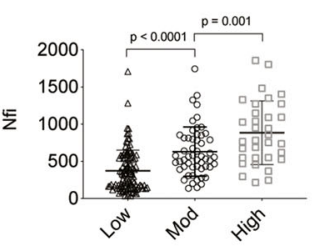

Endothelial lysate

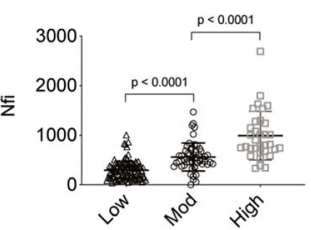

$\mathrm{Sm} / \mathrm{SmD}$

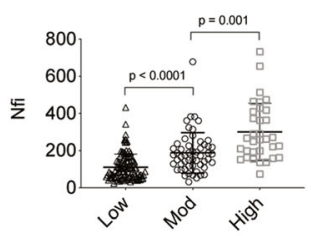

C

ANA among Low-High

d

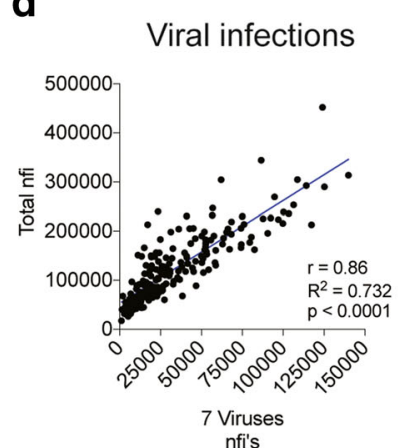

e

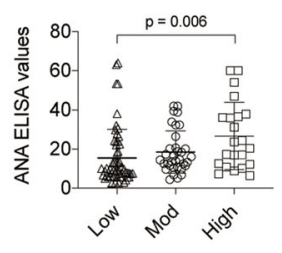

Odds ratio-High group

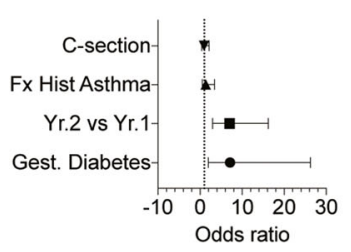

Fig. 4 Responses to selected autoantigens were greater in the high reactivity group a. The relative IgG responses (nfi values) are shown for diverse autoantigens among the low, Mod, and high groups. The high responder group exhibited a statistically significant increase in the IgG nfi values towards nucleosome components, arrestin b1, a histone mix, endothelial cell lysates, and the Smith antigens ( $\mathrm{Sm} / \mathrm{Smd})$. b Pearson's correlation analysis was performed for the sum of the total nfis for each of the 197 samples in relation to the summed nfis for 7 viruses (parvovirus, enterovirus 71, adenovirus type 5, RSV, Coxsackievirus, echovirus type 9, CMV), 5 live attenuated vaccines (mumps, rotavirus, rubella, rubeola, varicella-zoster), 7 subunit vaccines (hepatitis B, hepatitis A, Haemophilus influenzae, inactivated influenza types $\mathrm{A}$ and $\mathrm{B}$, tetanus toxoid, Streptococcus pneumoniae), and 8 autoantigens (arrestin beta 1, histones, myelin basic protein, myosin, endothelial lysates, Smith antigens, nucleosomes, alpha fodrin). Statistical analyses were done with Pearson correlation calculations using Graph Prism 8. The $r$ value and the $R^{2}$ values are indicated within the graphs. $\mathbf{c}$ The serum samples from a subset
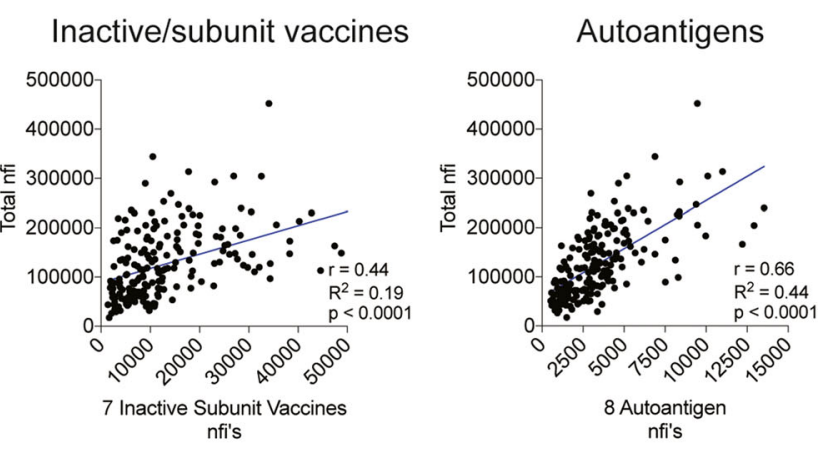

of toddlers (114/197) were used in ELISA assays to screen for the presence of anti-nuclear antibodies (ANA). The cutoff for positivity was defined by a previous analysis of over 700 samples from SLE patients, with positive controls provided within the ANA kit (ANA kit, QUANTA Lite ANA ELISA by Inova Diagnostics). Thirty (26\%) and eight (7\%) of these samples had moderate and high ANA titers. d The low, Mod, and high reactivity groups were compared for their ANA levels. Eight with high ANAs were part of the high reactivity group. e Odds ratio comparing the high responder cohort and diverse clinical indicators. The clinical information from 110 low and 33 high responder cohorts was compared to determine the relative odds ratio and statistical significance with particular clinical conditions. The strongest relationships exist with gestational diabetes followed by age. Statistical analyses were done with one-way ANOVA using Tukey's correlation, with the calculated $p$ values shown. ns, not significant. For odds ratio calculations, 2 -way calculations were performed with the $95 \%$ confidence intervals shown 
based clinical assay that provides standards to designate $\operatorname{IgG}$ titers as negative, low, and high to nuclear antigens. These were split into ANA negative, low and high (Fig. 4b). Surprisingly, $8 \%$ of the cohort had high ANA titers, with some individual's values approaching that seen in systemic lupus erythematosus patients (Fig. 4b, values $>50$ ). A statistically significant difference between the high and low cohorts (Fig. 4c). However, it was also obvious that several individuals in the low group also had high ANA titers. These results suggest that circulating ANA are already apparent in the healthy toddler/children population $[32,33]$.

Given the strong anti-viral nfi values noted among the high responder group, we assessed whether this was also true for responses to pathogens, vaccines, and autoantigens. The overall sum of the nfis for each individual was compared with the sum of the nfi values towards 7 common viral infections, 5 live attenuated vaccines, 7 inactivated and/or subunit vaccines, and 8 autoantigens (Fig. 4b). The highest positive correlation index existed between the overall sum of the nfis and viral responses (Fig. $4 \mathrm{~b}, r=0.86$ ). The 2 nd strongest positive correlation was with live attenuated vaccine responses $(r=0.78)$. Interestingly, the nfi values for different live attenuated vaccines were an order of magnitude less than those seen with the infectious agents. This suggests that pathogen responses may drive enhanced vaccine responses in the high response group. This was only found for live attenuated vaccines, as a relatively poor correlation existed in relation to inactive subunit vaccines (Fig. $4 \mathrm{~b}, r=0.44)$. Finally, a positive correlation was revealed in the high responder group and autoantigens (Fig. 4b, $r=$ $0.66)$.

The stratification of the toddlers/children into low, Mod, and high responder groups along with medical records allowed us to address whether these patterns correlated with particular clinical features. An odds ratio assessment was undertaken. There was a statistically significant odds ratio for the high responder cohort and maternal gestational diabetes followed by age (Fig. 4e). No increased odds ratios were noted for delivery type, family history of asthma or autoimmunity, C-section versus vaginal delivery, male versus female sex, nor up-to-date, or delayed vaccination status (Fig. 4e, data not shown). Some individual antigens for which male and female sex, C-section versus vaginal delivery, and age were statistically different could be found, but this was not based on our stratification strategy (Supplemental Table S3). Taken together, a comparison of the serum IgG antibody specificities in toddlers/children towards a diverse group of antigens reveals a cohort with elevated IgG nfi values towards common viruses. This elevated viral response may drive seropositive responses to live attenuated vaccines. This did not appear to be the case for inactive or subunit vaccines.

\section{Longitudinal Profiling of the Serum Antibody Specificities Suggest the High Response Cohort of Toddler/Children Maintain Their Responses}

Forty-one of the toddlers/children in our cohort had a 2nd-year follow-up blood draw, allowing a longitudinal assessment of their serum IgG changes (Fig. 5a, Supplemental Fig. 1b). Comparing similar groups of antigens as that shown in Figs. 2 and 3, the increased IgG nfi responses noted for specific viral pathogens: RSV, Coxsackievirus, echovirus type 9, and CMV grade 3 antigen were retained at Yr.2 for those having a response evident within their 1st year of life (Fig. $5 \mathrm{a}$, closed versus open circles). This was dependent on the pathogen, as most individuals exhibited only transient responses towards molluscum contagiosum, either evident at Yr.1 or Yr.2, but lost by Yr.2 if first uncovered in Yr.1. Certain individuals who had little IgG reactivity at Yr.1 seroconverted at Yr.2, confirming pathogen exposures and the transient nature of the circulating antibodies to particular viruses (Fig. 4a, red dotted lines connecting each individual). Another interesting finding from the profiling is that certain viral-specific antibody responses primarily occurred in the second year of life. This was evident for the RNA virus echovirus type 9 and two DNA viruses, EBV, and parvovirus (Fig. 5a).

In contrast to the infection-driven IgG nfi values in the tens of thousands, the IgG nfi values against live attenuated and inactive viruses along with subunit vaccines remained in the hundreds, regardless of whether this was a Yr.1 or Yr.2 sampling (Fig. 5a vs b). This is not unexpected, as infections drive higher antibody responses than vaccines. Among the toddlers, many individuals who had a low nfi value at Yr.1 maintained this low response at Yr.2 (Fig. 5b). All 41 individuals with repeat sampling had received their combined measles, mumps, rubella vaccine (MMR) along with those against rotavirus, varicella-zoster virus, and hepatitis A. Rotavirus is administered at 2, 4, and 6 months of age, while MMR is given at Yr.1 along with varicella and hepatitis A. Influenza vaccination is provided yearly at any point from 6 months on. Overall, these data suggest that each toddler/child generated an $\mathrm{IgG}$ response unique and distinct from others. A significant number of individuals who had low IgG binding responses in both their first and second years of life. This does not necessarily mean they have lack protective immune responses, as the live attenuated vaccines elicit effective $\mathrm{T}$ cell-mediated responses, which may provide sufficient protection.

\section{Genetic Association Analysis Show Enrichment of Genetic Risk Alleles in the High Responder Group}

Genome-wide association studies (GWASs) in several autoimmune, rheumatic, and immune system-related conditions in adults have uncovered several genetic variations that can 
a

Pathogens

RNA virus

RSV

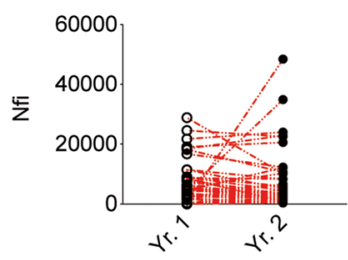

DNA virus

ADV type 5
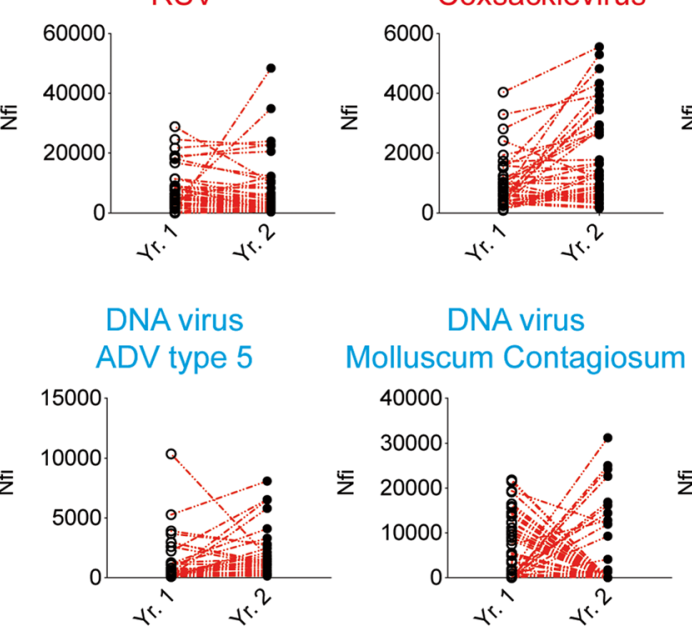

$z$

2000

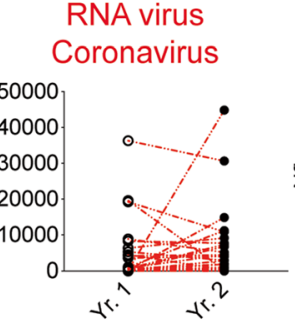

DNA virus

Epstein Barr
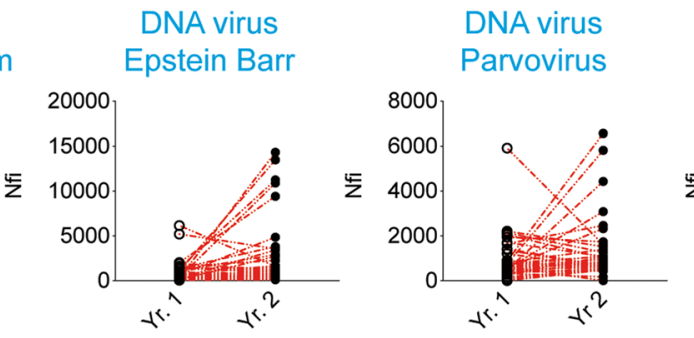

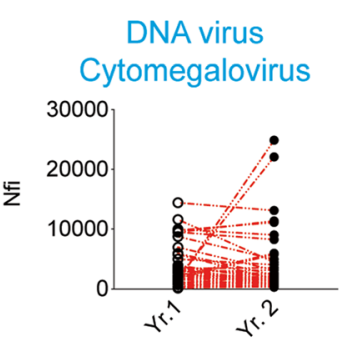

Bacteria

P. fluorescens (lipase)

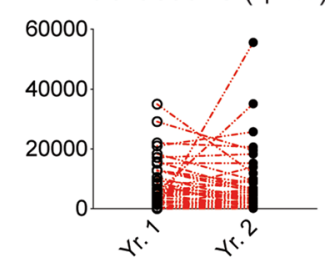

b

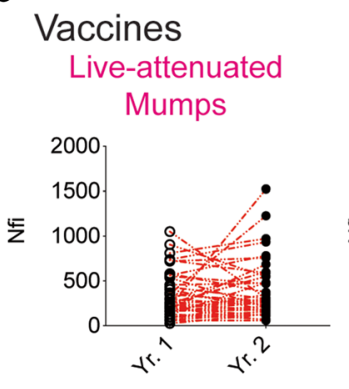

\section{Live-attenuated Rotavirus}

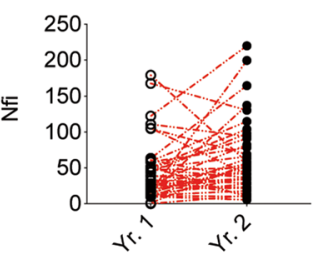

Subunit-virus Hepatitis B

Subunit-virus Hepatitis A

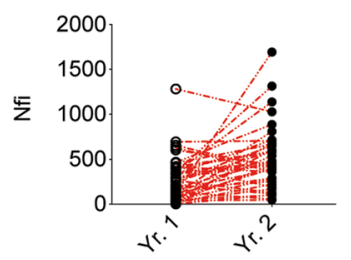

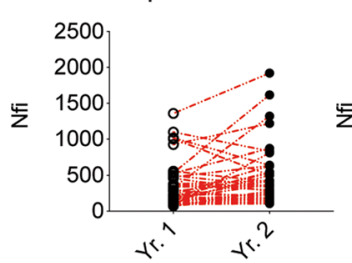

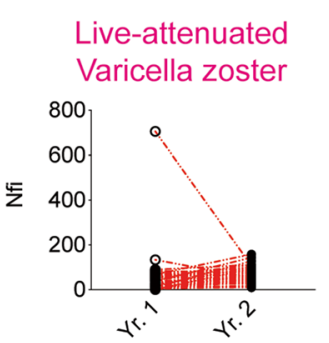

Subunit $H$. influenzae

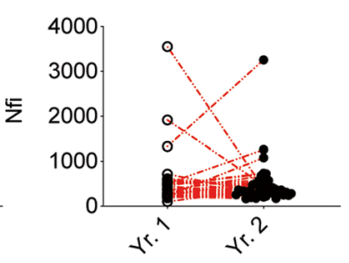

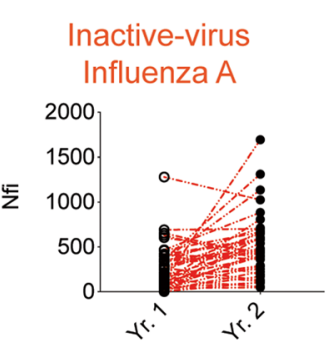

Subunit S. pneumoniae

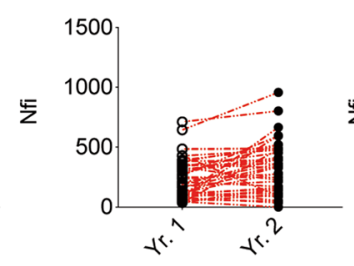

Inactive-virus Influenza B

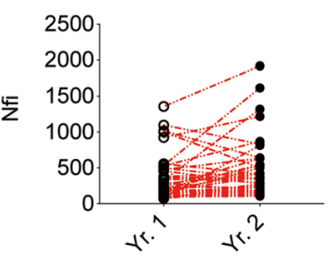

Subunit

Tetanus toxoid

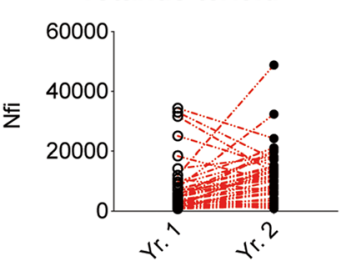

Fig. 5 Longitudinal profiling of serum IgG nfi responses in toddlers/ children from year 1 to year 2 reveals both fixed and fluctuating patterns dependent on the antigen. a The relative IgG responses (nfi values) are shown against various RNA viruses (red), DNA viruses (blue), and a bacterial antigen (black) as indicated. These values are shown for 41 repeat samples, using a serum sample from Yr.1 followed by a second sample at Yr.2. The high responder group exhibited a statistically

significant increase in the IgG nfi values towards selected viral pathogen antigens, as shown. This included responses to RSV, Coxsackievirus, echovirus type 9, CMV, and adenovirus type 5. b The relative IgG responses to either live attenuated, inactivated, or subunit vaccines were determined with the 41 repeats. Statistical analyses were done with oneway ANOVA using Tukey's correlation, with the calculated $p$ values shown. ns, not significant

contribute to disease susceptibility [34-37]. To assess the prevalence of such previously identified genetic risk alleles, we genotyped 114 samples using an Infinium Global Screening Array-24 v3.0 BeadChip from Illumina. These 114 samples were selected based on various criteria such as availability of at least $250 \mathrm{ng}$ of QC pass genomic DNA and completeness of immune profiling data. Genotyping array used in the study combines multi-ethnic genome-wide content, curated clinical research variants, and quality control (QC) markers and about two hundred thousand immune- related genetic variants, including 345 potential immune regulatory variants (Fig. 6a). These were selected based on their previous mapping with autoimmune patients versus normal individuals [38, 39].

To assess the effect of carrying multiple genetic risk alleles in immune response genes in relation to the magnitude of the IgG response, a genetic risk score (GRS) was generated for each individual based on the presence or absence of 47 top risk alleles that were found associated with nfis in a quantitative association test (Fig. 6a, Supplemental Table S4). These 
a

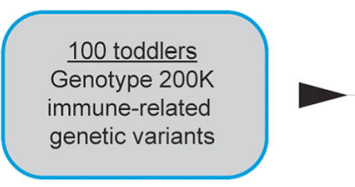

b

Risk alleles/group

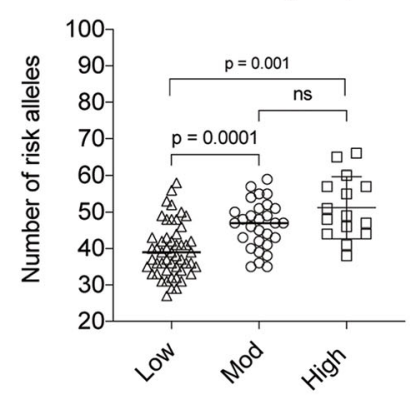

e

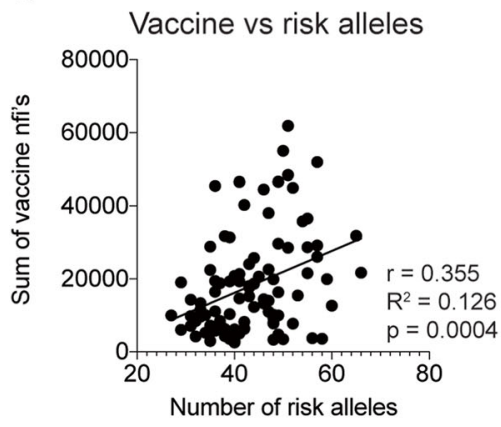

SNP array analysis pipeline

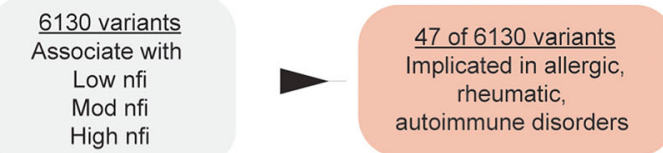

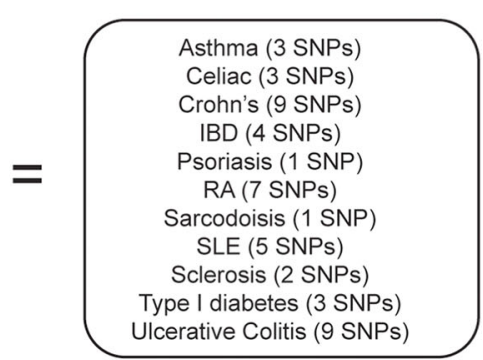

d

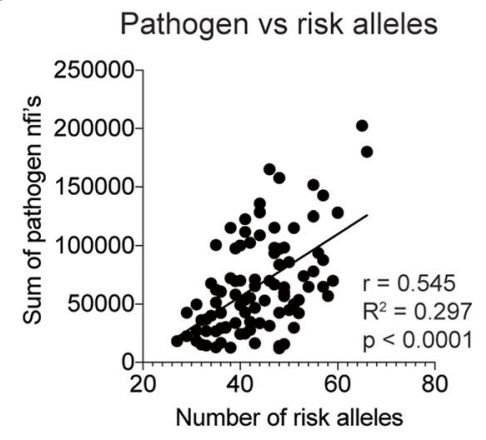

g

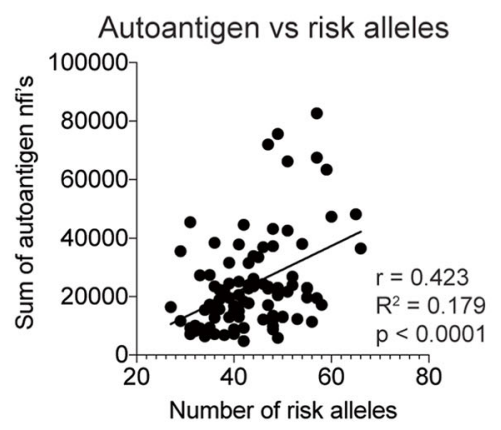

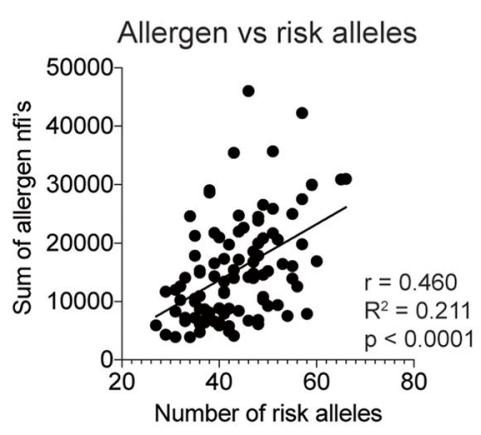

h

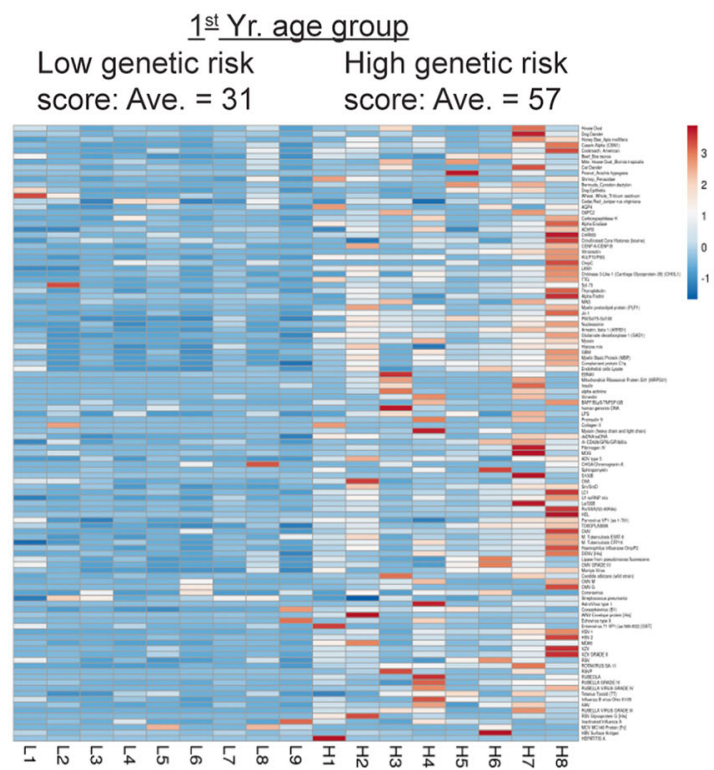

variants have been implicated in various autoimmune, rheumatic, and allergic conditions previously (Fig. 6a). This

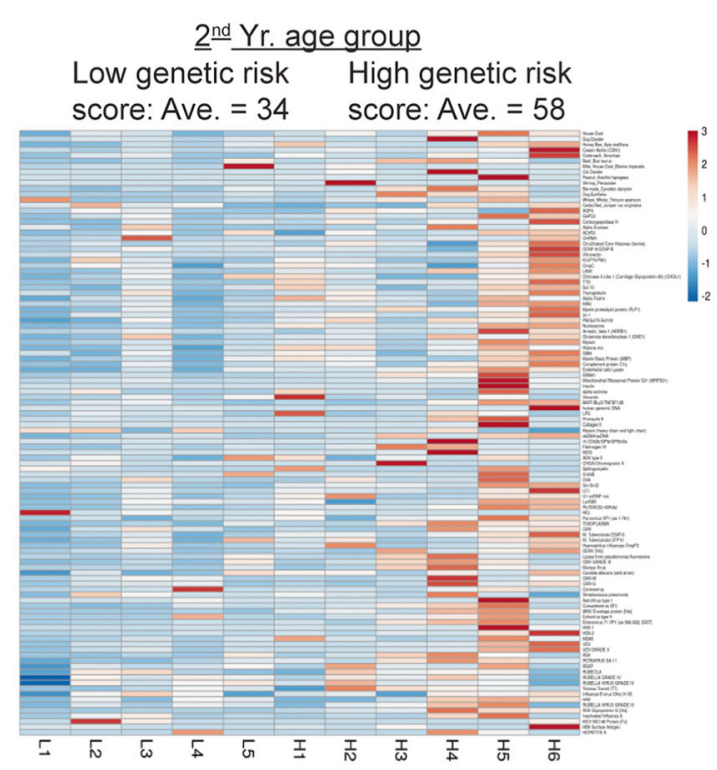

analysis was developed based on earlier work defining genetic risk alleles in the setting of SLE [38, 39]. A statistically 
Fig. 6 Correlation between genetic risk alleles and high responder cohort. a Schematic approach for identifying SNPs in immune response genes in the toddlers/children that have been linked to various clinical conditions including asthma, celiac, Crohn's disease, inflammatory bowel disease (IBD), rheumatoid arthritis (RA), lupus (SLE), sclerosis, type I diabetes, and ulcerative colitis. b The sum total of each of the 47 genetic risk alleles (94 total when considering both alleles) was determined for 114 individual toddlers. These values were plotted against the low $(n=$ $110)$, Mod $(n=54)$, and high $(n=33)$ responder cohorts. c Pearson's correlation analysis was performed for the sum of the total genetic risk alleles for 114 samples in relation to the summed IgG nfis towards the 110 distinct antigens. Statistical analysis was done by Pearson's correlation. The $r$ value and the $R^{2}$ values are indicated within the graph. $\mathbf{d}$ The low and high genetic risk scores were compared within the 1st year age group and the 2 nd year age group

significant accumulation of risk alleles in multiple genes was observed for the Mod and high versus low response group (Fig. 6b, $p<0.0001$ ). This was further supported by a high Pearson's correlation $(r=0.61, p<0.001)$ between number of risk alleles and the sum nfi values (Fig. 6c). To determine if a combination of age and genetic risk scores impacted the antibody responses to the 110 antigens, the two age groups were segregated and a subset of individuals in the low GRS versus high GRS was compared with the serum IgG responses (Fig. 6d). Both Yr.1 and Yr.2 cohorts with high GRS had positive IgG responses to multiple antigens on the protein array relative to those in the low GRS. Yet, the numbers did not differ between Yr.1 and Yr.2, revealing that the genetic risk allele correlation with IgG reactivity was independent of age. In addition, there was a strong correlation found with a higher GRS score and anti-pathogen as well as autoantigen responses. No statistically significant correlations were found with vaccine immune responses.

Of the 47 top allelic variants, the rs2284033 SNP in ILR2B is a known genome-wide association studies (GWAS) variant linked to juvenile idiopathic arthritis and asthma [38, 39]. According to GTEx (Genotype-Tissue Expression database (https://gtexportal.org)), this SNP is an eQTL, with the disease-associated allele $(\mathrm{G})$ having a higher expression of the $I L 2 R B$ gene in multiple cell lineages. To determine if there was a correlation among the high versus low responder in relation to particular disease risk alleles, we further studied two individuals in the high response group (147.1 and 72.1, red circles) and two in the low response group $(132.1,141.1)$. The two in the high response group had the $\mathrm{G} / \mathrm{G}$ genotype for rs2284033, while two in the low cohort had an A/G (Fig. 7a, blue). Further review of their medical history revealed that both these individuals in the high group had asthma. We selected a second gene, TNFSF4, in which the G/T and T/T variants (rs7514229) are implicated in SLE. The T/T genotype results in a higher expression of TNFSF4 (https://gtexportal. org). As shown, the high and low response subjects had the moderate $(\mathrm{G} / \mathrm{T})$ and high-risk genotypes $\mathrm{T} / \mathrm{T}$ suggesting this marker did not segregate the two groups (Fig. 7b). Finally, we compared rs689, a known risk allele in the insulin (IGF2) gene for autoimmune or type 1 diabetes [40]. While the T/T allele was more prevalent in the high responder group, as seen in the two high responders $(72.1,141.1)$, one of the low responders had the T/T allele (Fig. 7c). These findings suggest a compilation of different risk alleles among a larger cohort will be needed to ascertain the direct contributions of risk alleles on the overall antibody responses.

To examine the association between the IL2RB, TNFSF4, and $I G F 2$ risk alleles and serum cytokine/chemokine responses, we compared the levels of these in the same four individuals described above. As shown in the heat map in Fig. 7d, several cytokines and chemokines were significantly higher in high response individuals (147.1 and 72.1) versus those in the low response group (132.1 and 141.1) (Fig. 7d). Interestingly, both 147.1 and 72.1 also had elevated nfis of clinical significance against CMV and EBV, while 132.1 and 141.1 did not. These results provide a proof-of-concept of our approach to identify subjects with potential for disease risk. Such pilot findings suggest that a genetic risk profile of all individuals in our study may provide additional insights into the influence of genetic susceptibility alleles on the serum IgG responses against various pathogens and consequent outcomes for autoantibody responses. In future directions, we will use this strategy to compare more individuals with a higher risk profile in relation to their cytokine/chemokine levels.

\section{Discussion}

Toddlers/children generate specific antibodies in response to pathogen exposures, the microbiome, environmental antigens, and vaccinations. While many studies have examined antibody responses to both infectious and vaccine responses in babies, these reports have focused primarily on specific antigens related to the infection or vaccine under query [41, 42]. In the current study, we compared the serum antibody specificities to 110 distinct antigens among a cohort of 1- and 2year-old toddlers/children.

The toddlers, seen during their standard of care wellness visits at Yr.1 and Yr.2, were found to diverge markedly in the magnitude and nature of their serum IgG responses. One subgroup, designated as high responders, exhibited statistically significant elevated IgG nfis to numerous RNA and DNA viruses compared to the Mod and low response groups. These elevated seropositive responses targeting 7 different viruses correlated with higher nfi values against live attenuated viral vaccines and, surprisingly, autoantigens. Enhanced $\mathrm{IgG}$ reactivities to various infection agents are expected given the frequent exposures toddlers have to respiratory syncytial virus (RSV), Coxsackievirus, cytomegalovirus, molluscum contagiosum, echovirus type 9 , and others. RSV is the most 
a

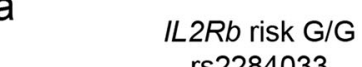

rs2284033

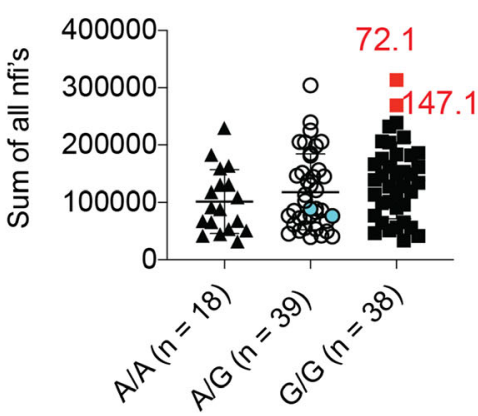

b TNFSF4 risk A/G,G/G
rs7514229

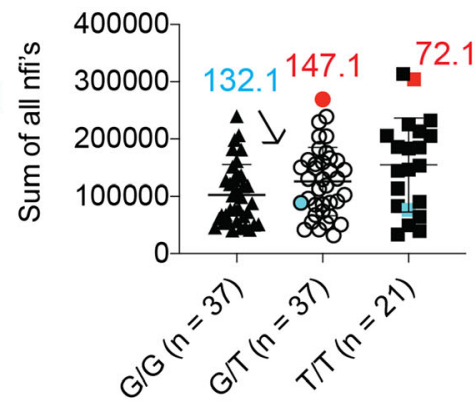

C IGF2 risk $\mathrm{T} / \mathrm{T}$ rs689

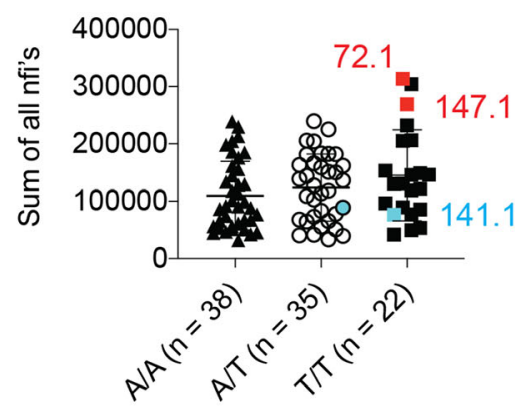

d Serum cytokine levels
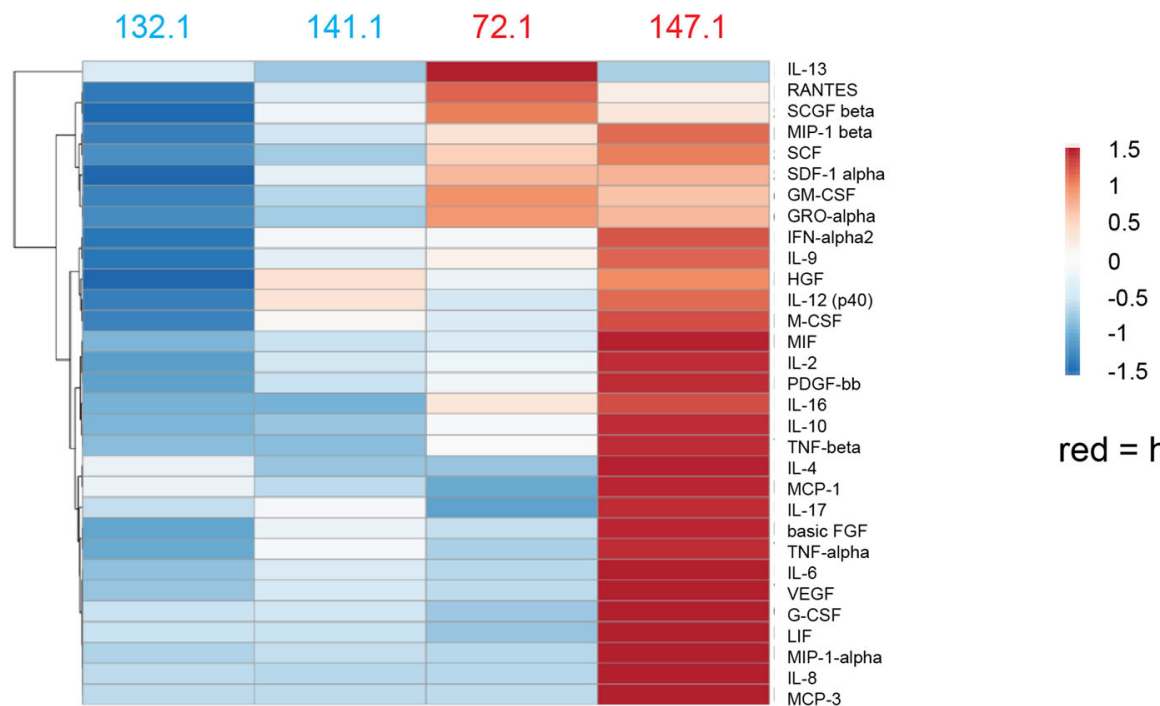

red $=$ high

Fig. 7 DNA sequence polymorphisms in immune responses genes linked to elevated serum cytokine levels in toddlers. a-c The total sum of the IgG nfi responses for every antigen was calculated. These values were plotted against the allelic polymorphisms in the cohort for the genes: a IL2 receptor beta gene (rs2284033), b TNFSF4 (rs7514229), and c IGF2 (rs689). Two of the toddlers in the high response group (72.1 and 147.1) are indicated with the red colored circles or boxes. The two from the low response group $(131.1,141.1)$ are in blue. d Four serum samples, two from the IL2rb (rs2284033) A/G haplotype $(132.1,141.1)$ and two from G/G genotype (147.1, 72.1), were analyzed with the Human 48 Plex BioRad cytokine/chemokine assay. A heat map was used to illustrate the levels of inflammatory cytokines and chemokines present in each sample. Two individuals, who are in the high responder categories, had markedly elevated levels of the indicated cytokines $(147.1,72.1)$ common cause of viral lower respiratory tract infection (bronchiolitis) in infants [43]. Elevated nfis in the high response cohort were not against all viruses, as the seropositivity to certain CMV antigens (CMV and CVM-M), coronavirus, and molluscum contagiosum was similar among the high, Mod, and low responder group. Cytomegalovirus (CMV), a herpes virus family member, has a pronounced impact on the emerging immune repertoire [44]. While usually asymptomatic in healthy subjects, it causes disease in immunocompromised individuals. The elevated IgG responses towards CMV targeted two distinct antigens, CMV and CMV-M, with seropositivity confirmed by an ELISA assay. Yet, the high responder cohort had a statistically significantly higher IgG response to
CMV grade III antigen relative to the low and Mod response groups. Seropositive responses to this antigen are proposed to reflect a period of active viral replication (Supplemental Fig. 3) [30]. We found this response correlated with increased IgG responses to autoantigens. A second unexpected result concerns the absence of a correlation between the microfluidic nfis to the EBV EBNA1 antigen relative to the ELISA assay. While some seropositivity using the arrays and ELISA overlapped, there were some seropositive signals in the array that were negative with the ELISA and vice-versa (Supplemental Fig. 2c). To address this discrepancy, the EBNA1 antigens used by different vendors were compared. This revealed that the full-length protein is not typically used in any of the assays, and instead, 
different fusion proteins comprising various segments of EBNA1 are used by the manufacturers. Thus, a single kit may not reveal all seropositive responses. Second, comparing our antigens and those from the ELISA kit reveals that the actual region in EBNA1 targeted by an antibody varies among the population. To identify all toddlers with EBV seropositivity in the future, multiple overlapping EBNA1 antigens will need to be incorporated into the microfluidic array. IgG responses to molluscum contagiosum, a poxvirus, were also found to be independent of the toddler low, Mod, and high stratification (Fig. 3a) [45]. For this virus, seropositive responses appeared to be very transient. Individuals with seroreactivity at Yr.1 lost these titers by Yr.2 post-infection. We also identified 3 children with seropositive responses to the West Nile virus, a mosquito-borne pathogen evident in North Texas. Finally, 3 individuals had $\mathrm{IgG}$ nfi values suggestive of responses to the Mycobacterium tuberculosis proteins, ESAT-6, and culture filtrate protein 10 (CFP-10), which are part of the QuantiFERON TB Gold-based diagnostic tests for tuberculosis [46]. Each toddler had tuberculosis risk factors. Two were born in Africa and the third one had a trip to Mexico. Only one subject was positive for the tuberculin purified protein derivative (PPD) test and this toddler had the highest IgG values against ESAT- 6 and CFP-10 antigens.

While most antibodies are considered protective, some can target self-antigens such as proteins, nucleic acids, complex carbohydrates, and lipids. This can lead to an autoimmune disease, with antibodies attacking and destroying normal cells and tissues in the body. An unanticipated finding in our study was the increased IgG nfi responses to various autoantigens in the high responder cohort comprising both 1- and 2-year-old age groups. In fact, the nfi IgG values in the high cohort targeting several distinct autoantigens reached clinically relevant levels based on a comparison with a cohort of adult lupus patients. Several autoimmune diseases in children have been reported, including juvenile idiopathic arthritis, juvenile dermatomyositis, autoimmune thrombocytopenia, and proctitis-proctocolitis (food allergen response) [47-49]. However, the cohort in our study did not have any indication of such autoimmune presentations. In addition, autoimmune diseases in children are typically not reported for 1-2 years olds [38, 50-54]. To further explore the autoimmune responses, ANA ELISAs were done. ANA titers of clinical significance were discovered in toddlers in both the low and high response groups. Twenty to $30 \%$ of healthy adults can produce ANA without any clinical signs of autoimmune diseases $[50,51]$. Several viruses are proposed to trigger autoimmune diseases, including CMV, Coxsackievirus type B, EBV, and herpes simplex virus (HSV) [31, 55]. EBV has been associated with the development of SLE while systemic sclerosis has been linked to CMV and parvovirus. CMV has been detected in patients with rheumatoid arthritis, Sjogren's syndrome, polydermatomyositis, psoriasis, Wegener's granulomatosis, and Crohn's disease. However, others have suggested that such infections and exposures protect against autoimmunity
$[56,57]$. By continuing our longitudinal profiling at Yrs. 4 and 8 , we will be able to better address this question. This may benefit from the inclusion of a SNP array analysis as we described for 4 individuals from our study. For this, a selected subset of SNPs was screened based on their strong link to autoimmune and inflammatory responses. This analysis uncovered a positive correlation between the sum of the "disease"linked SNPs (genetic risk score) and the elevated nfis of the high response group. Our data does suggest that longitudinal monitoring of clinical signs and antibody profiles in the subset that has an enrichment of previously known genetic risk alleles may be a potential strategy to identify individuals with increased risk and to offer a timely diagnosis. As part of our study, we included different allergens (Supplemental Fig. 3c). When analyzing the antibody responses to these allergens, only $\operatorname{IgG}$ values were measured. The high responder group had elevated IgG nfi values to beef antigens (Supplemental Fig. 3c). A more effective strategy to assess the allergen response is to measure the serum IgE values, which can be performed using the microfluidic antigen arrays. In future directions, we will examine the IgE levels with a clinically defined cohort of asthmatic/allergic patients versus healthy controls.

It was interesting to discover that a substantial number of toddlers maintained relatively low IgG reactivities to the antigens listed in the array into the second year of life. Clinically, such low responses have been reported in toddlers with transient hypogammaglobulinemia of infancy, which can extend into the 3 rd year of life. However, the cohort in our study had normal IgG and IgM antibody levels. This again supports the overall idea that inherent variations exist among the human population in the capacity of individuals to respond to infections. Findings from our study may identify toddlers at risk for immune system abnormalities. The key advantages of the microfluidic antigen array designed for toddlers/children are the small serum volumes needed, the ability to monitor IgG responses to diverse antigens simultaneously, the identification of individuals with previously unrecognized infections, and the realization that the antibody responses are very divergent among different similar-aged toddlers. As such, this toddler serum IgM and IgG profiling screen may also be very beneficial in assessing the responses for patients with different inborn errors of immunity[58-60]. The array may facilitate assessments of the serum antibody changes during the COVID-19 pandemic. We recently developed microfluidic antigen arrays to include numerous coronavirus antigens and used these with an X-linked severe combined immunodeficient infant who became infected with SARS-CoV-2 [61].

Supplementary Information The online version contains supplementary material available at https://doi.org/10.1007/s10875-021-00993-w.

Acknowledgments We sincerely appreciate the pediatricians who permitted our consenting of their patients/families. These included Dr. Nancy Kelly, who was the first clinician to initiate this study with us, 
and Dr. Dawn Johnson, current director of the Children's Health Specialty Center, Dallas, TX. We thank Dr. Pratibha Bhalla and Mr. Austin Thompson for technical support.

Author Contributions P.P-R., P.R., Q-Z.L., and N.S.C.v.O. conceived the experiments. M.N., P.P-R., and N.S.C.v.O. consented families and collected the blood samples. R.S.G. provided information about clinical presentation including asthma and allergy. P.P-R, P.R, Q-Z Li, and N.S.C.v.O. performed the experiments. P.P-R, D. K, R.G., and N.S.C.v.O. reviewed the clinical information. P.P-R, D. K, I.D., R.G., E.K.W., M.d.1.M., and N.S.C.v.O. analyzed and discussed the data. P.PR, P.R., Q-Z. Li, and N.S.C.v.O. wrote the manuscript.

Funding This work was supported, in part, by grants from the National Institutes of Health R01 (R01 AI1 14523 N.S.C.v.O.) and Beecherl funds from the Department of Immunology at UT Southwestern Medical Center (N.S.C.v.O.).

Data Availability All data generated or analyzed during this study are included in this published article. The most detailed data report from which the figures were developed is provided as a Supplemental Excel spreadsheet. The Kid Panel I arrays are available from the Microarray and Immunophenotyping Core at UT Southwestern Medical Center. The array data from our study are included in Supplemental information.

\section{Declarations}

Consent to Participate This research involved human subjects. The study was performed in accordance with the ethical standards as laid down in the 1964 Declaration of Helsinki and its later amendments. Parental consent was obtained for 295 toddlers, of who 197 were used for the analyses in the current study. The Institutional Review Board at UT Southwestern Medical Center approved this study (IRB\# 072010-009 and IRB\# 112010-013).

Consent to Publish The patients presented in the study are de-identified. All families were consented to participate in the study and had knowledge that the outcome of the findings would be published in a scientific journal.

Conflict of Interest The authors declare no conflict of interest

\section{References}

1. Olin A, Henckel E, Chen Y, Lakshmikanth T, Pou C, Mikes J, et al. Stereotypic immune system development in newborn children. Cell. 2018;174(5):1277-687538176.

2. Chen Y, Chaudhary N, Yang N, Granato A, Turner JA, Howard SL, et al. Microbial symbionts regulate the primary Ig repertoire. J Exp Med. 2018;215(5):1397-415.

3. de Jong SE, Olin A, Pulendran B. The impact of the microbiome on immunity to vaccination in humans. Cell Host Microbe. 2020;28(2):169-79.

4. Ygberg S, Nilsson A. The developing immune system - from foetus to toddler. Acta Paediatr. 2012;101(2):120-7.

5. Griffin DO, Holodick NE, Rothstein TL. Human B1 cells in umbilical cord and adult peripheral blood express the novel phenotype CD20+CD27+CD43+CD70-. J Exp Med. 2011;208(1):67-80.

6. Quách TD, Rodríguez-Zhurbenko N, Hopkins TJ, Guo X, Hernández AM, Li W, et al. Distinctions among circulating antibody-secreting cell populations, including B-1 cells, in human adult peripheral blood. J Immunol. 2016;196(3):1060-9.
7. de Vries E, de Bruin-Versteeg S, Comans-Bitter WM, de Groot R, Hop WCJ, Boerma GJM, et al. Longitudinal survey of lymphocyte subpopulations in the first year of life. Pediatr Res. 2000;47(4): 528-37.

8. Morbach H, Eichhorn EM, Liese JG, Girschick HJ. Reference values for B cell subpopulations from infancy to adulthood. Clin Exp Immunol. 2010;162(2):271-9.

9. Simon AK, Hollander GA, McMichael A. Evolution of the immune system in humans from infancy to old age. Proc R Soc B Biol Sci. 2015;282(1821):20143085.

10. Goo L, Chohan V, Nduati R, Overbaugh J. Early development of broad neutralizing antibodies in HIV-1 infected infants. Nat Med. 2014;20(6):655-8.

11. Wendel BS, He C, Qu M, Wu D, Hernandez SM, Ma K-Y, et al. Accurate immune repertoire sequencing reveals malaria infection driven antibody lineage diversification in young children. Nat Commun. 2017;8(1):531.

12. Siegrist C-A. Neonatal and early life vaccinology. Vaccine. 2001;19(25):3331-46.

13. Niewiesk S. Maternal antibodies: clinical significance, mechanism of interference with immune responses, and possible vaccination strategies. Front Immunol. 2014;5:446.

14. Edwards KM. Maternal antibodies and infant immune responses to vaccines. Vaccine. 2015;33(47):6469-72.

15. $\mathrm{Hu} \mathrm{Y,} \mathrm{Wu} \mathrm{Q,} \mathrm{Xu} \mathrm{B,} \mathrm{Zhou} \mathrm{Z,} \mathrm{Wang} \mathrm{Z,} \mathrm{Zhou} \mathrm{YH.} \mathrm{Influence} \mathrm{of}$ maternal antibody against hepatitis $B$ surface antigen on active immune response to hepatitis $B$ vaccine in infants. Vaccine. 2008;26(48):6064-7.

16. Kılıc A, Altınkaynak S, Ertekin V, Inandı T. The duration of maternal measles antibodies in children. J Trop Pediatr. 2003;49(5): 302-5.

17. Ochola R, Sande C, Fegan G, Scott PD, Medley GF, Cane PA, et al. The level and duration of RSV-specific maternal IgG in infants in Kilifi Kenya. PLoS One. 2009;4(12):e8088.

18. Dorsey MJ, Dvorak CC, Cowan MJ, Puck JM. Treatment of infants identified as having severe combined immunodeficiency by means of newborn screening. J Allergy Clin Immunol. 2017;139(3):73342.

19. Bousfiha A, Jeddane L, Picard C, Al-Herz W, Ailal F, Chatila T, et al. Human inborn errors of immunity: 2019 update of the IUIS phenotypical classification. J Clin Immunol. 2020;40(1):66-81.

20. Dixit CK, Aguirre GR. Protein microarrays with novel microfluidic methods: current advances. Microarrays (Basel). 2014;3(3):180 202.

21. Li QZ, Xie C, Wu T, Mackay M, Aranow C, Putterman C, et al. Identification of autoantibody clusters that best predict lupus disease activity using glomerular proteome arrays. J Clin Invest. 2005; 115(12):3428-39.

22. Li Q-Z, Zhou J, Wandstrat AE, Carr-Johnson F, Branch V, Karp DR, et al. Protein array autoantibody profiles for insights into systemic lupus erythematosus and incomplete lupus syndromes. Clin Exp Immunol. 2007;147(1):60-70.

23. Li QZ, Zhou J, Wandstrat AE, Carr-Johnson F, Branch V, Karp $\mathrm{DR}$, et al. Protein array autoantibody profiles for insights into systemic lupus erythematosus and incomplete lupus syndromes. Clin Exp Immunol. 2007;147(1):60-70.

24. Dozmorov I, Saban MR, Knowlton N, Centola M, Saban R. Connective molecular pathways of experimental bladder inflammation. Physiol Genomics. 2003;15(3):209-22.

25. Dozmorov I, Centola M. An associative analysis of gene expression array data. Bioinformatics. 2003;19(2):204-11.

26. Dozmorov I, Knowlton N, Tang Y, Centola M. Statistical monitoring of weak spots for improvement of normalization and ratio estimates in microarrays. BMC Bioinformatics. 2004;5:53. 
27. Dozmorov I, Lefkovits I. Internal standard-based analysis of microarray data. Part 1: analysis of differential gene expressions. Nucleic Acids Res. 2009;37(19):6323-39.

28. Dozmorov IM, Jarvis J, Saban R, Benbrook DM, Wakeland E, Aksentijevich I, et al. Internal standard-based analysis of microarray data 2-analysis of functional associations between HVE-genes. Nucleic Acids Res. 2011;39(18):7881-99.

29. Zhu H, Luo H, Yan M, Zuo X, Li Q-Z. Autoantigen microarray for high-throughput autoantibody profiling in systemic lupus erythematosus. Genomics Proteomics Bioinformatics. 2015;13(4):210-8.

30. Breda G, Almeida B, Carstensen S, Bonfim CM, Nogueira MB, Vidal LR, et al. Human cytomegalovirus detection by real-time PCR and pp65-antigen test in hematopoietic stem cell transplant recipients: a challenge in low and middle-income countries. Pathog Glob Health. 2013;107(6):312-9.

31. Temajo NO, Howard N. The mosaic of environment involvement in autoimmunity: the abrogation of viral latency by stress, a noninfectious environmental agent, is an intrinsic prerequisite prelude before viruses can rank as infectious environmental agents that trigger autoimmune diseases. Autoimmun Rev. 2014;13(6):63540.

32. Soto ME, Hernández-Becerril N, Perez-Chiney AC, HernándezRizo A, Telich-Tarriba JE, Juárez-Orozco LE, et al. Predictive value of antinuclear antibodies in autoimmune diseases classified by clinical criteria: analytical study in a specialized health institute, one year follow-up. Res Immunol. 2013;5:13-22.

33. Satoh M, Chan EKL, Ho LA, Rose KM, Parks CG, Cohn RD, et al. Prevalence and sociodemographic correlates of antinuclear antibodies in the United States. Arthritis Rheum. 2012;64(7):2319-27.

34. Gutierrez-Arcelus M, Rich SS, Raychaudhuri S. Autoimmune diseases - connecting risk alleles with molecular traits of the immune system. Nat Rev Genet. 2016;17(3):160-74.

35. Gateva V, Sandling JK, Hom G, Taylor KE, Chung SA, Sun X, et al. A large-scale replication study identifies TNIP1, PRDM1, JAZF1, UHRF1BP1 and IL10 as risk loci for systemic lupus erythematosus. Nat Genet. 2009;41(11):1228-33.

36. Wakeland EK, Liu K, Graham RR, Behrens TW. Delineating the genetic basis of systemic lupus erythematosus. Immunity. 2001;15(3):397-408.

37. Wandstrat AE, Nguyen C, Limaye N, Chan AY, Subramanian S, Tian $\mathrm{XH}$, et al. Association of extensive polymorphisms in the SLAM/CD2 gene cluster with murine lupus. Immunity. 2004;21(6):769-80.

38. Raj P, Song R, Zhu H, Riediger L, Jun D-J, Liang C, et al. Deep sequencing reveals a DAP1 regulatory haplotype that potentiates autoimmunity in systemic lupus erythematosus. Genome Biology. 2020;21(1):281.

39. Raj P, Rai E, Song R, Khan S, Wakeland BE, Viswanathan K, et al. Regulatory polymorphisms modulate the expression of HLA class II molecules and promote autoimmunity. eLife. 2016;5:e12089.

40. Onengut-Gumuscu S, Chen W-M, Burren O, Cooper NJ, Quinlan AR, Mychaleckyj JC, et al. Fine mapping of type 1 diabetes susceptibility loci and evidence for colocalization of causal variants with lymphoid gene enhancers. Nat Genet. 2015;47(4):381-6.

41. Esposito S, Scarselli E, Lelii M, Scala A, Vitelli A, Capone S, et al. Antibody response to respiratory syncytial virus infection in children $<18$ months old. Hum Vaccin Immunother. 2016;12(7):17006.

42. Dollard SC, Keyserling H, Radford K, Amin MM, Stowell J, Winter J, et al. Cytomegalovirus viral and antibody correlates in young children. BMC Res Notes. 2014;7:776.
43. Stein RT, Bont LJ, Zar H, Polack FP, Park C, Claxton A, et al. Respiratory syncytial virus hospitalization and mortality: systematic review and meta-analysis. Pediatr Pulmonol. 2017;52(4):556-69.

44. Brodin P, Jojic V, Gao T, Bhattacharya S, Angel CJL, Furman D, et al. Variation in the human immune system is largely driven by non-heritable influences. Cell. 2015;160(1-2):37-47.

45. Chen X, Anstey AV, Bugert JJ. Molluscum contagiosum virus infection. Lancet Infect Dis. 2013;13(10):877-88.

46. Tripathi DK, Srivastava K, Nagpal KL, Shukla PK, Srivastava KK. Exploration of some new secretory proteins to be employed for companion diagnosis of mycobacterium tuberculosis. Immunol Lett. 2019;209:67-74.

47. Sekerkova A, Fuchs M, Cecrdlova E, Svachova V, Kralova Lesna I, Striz I, et al. High prevalence of neutrophil cytoplasmic autoantibodies in infants with food protein-induced proctitis/proctocolitis: autoimmunity involvement? J Immunol Res. 2015;2015:902863.

48. Martin N, Li CK, Wedderburn LR. Juvenile dermatomyositis: new insights and new treatment strategies. Ther Adv Musculoskelet Dis. 2012;4(1):41-50.

49. Cepika A-M, Banchereau R, Segura E, Ohouo M, Cantarel B, Goller K, et al. A multidimensional blood stimulation assay reveals immune alterations underlying systemic juvenile idiopathic arthritis. J Exp Med. 2017;214(11):3449-66.

50. Wandstrat AE, Carr-Johnson F, Branch V, Gray H, Fairhurst AM, Reimold A, et al. Autoantibody profiling to identify individuals at risk for systemic lupus erythematosus. J Autoimmun. 2006;27(3): $153-60$.

51. Arbuckle MR, McClain MT, Rubertone MV, Scofield RH, Dennis GJ, James JA, et al. Development of autoantibodies before the clinical onset of systemic lupus erythematosus. N Engl J Med. 2003;349(16):1526-33.

52. Steiner G, Smolen J. Autoantibodies in rheumatoid arthritis and their clinical significance. Arthritis Res. 2002;4(Suppl 2):S1-5.

53. Chung L, Utz PJ. Antibodies in scleroderma: direct pathogenicity and phenotypic associations. Curr Rheumatol Rep. 2004;6(2):15663.

54. Martini A, Lorini R, Zanaboni D, Ravelli A, Burgio RG. Frequency of autoantibodies in normal children. Am J Dis Child. 1989;143(4): 493-6.

55. Molina V, Shoenfeld Y. Infection, vaccines and other environmental triggers of autoimmunity. Autoimmunity. 2005;38(3):235-45.

56. Gaisford W, Cooke A. Can infections protect against autoimmunity? Curr Opin Rheumatol. 2009;21(4):391-6.

57. Bach JF. Protective role of infections and vaccinations on autoimmune diseases. J Autoimmun. 2001;16(3):347-53.

58. Amaya-Uribe L, Rojas M, Azizi G, Anaya JM, Gershwin ME. Primary immunodeficiency and autoimmunity: a comprehensive review. J Autoimmun. 2019;99:52-72.

59. Todoric K, Koontz JB, Mattox D, Tarrant TK. Autoimmunity in immunodeficiency. Curr Allergy Asthma Rep. 2013;13(4):361-70.

60. Sullivan KE. Chromosome 22q11.2 deletion syndrome and DiGeorge syndrome. Immunol Rev. 2019;287(1):186-201.

61. van Oers NSC, Hanners NW, Sue PK, Aquino V, Li Q-Z, Schoggins JW, et al. SARS-CoV-2 infection associated with hepatitis in an infant with X-linked severe combined immunodeficiency. Clin Immunol. 2021;224:108662.

Publisher's Note Springer Nature remains neutral with regard to jurisdictional claims in published maps and institutional affiliations. 